\title{
A COMPARATIVE STUDY OF DENSITY FIELD ESTIMATION FOR GALAXIES: NEW INSIGHTS INTO THE EVOLUTION OF GALAXIES WITH ENVIRONMENT IN COSMOS OUT TO $z \sim 3$
}

\author{
Behnam Darvish ${ }^{1}$, Bahram Mobasher ${ }^{1}$, David Sobral ${ }^{2,3,4}$, Nicholas Scoville ${ }^{5}$, and Miguel Aragon-Calvo ${ }^{1}$ \\ ${ }^{1}$ University of California, Riverside, 900 University Ave, Riverside, CA 92521, USA; bdarv001@ucr.edu \\ ${ }^{2}$ Instituto de Astrofísica e Ciências do Espaço, Universidade de Lisboa, OAL, Tapada da Ajuda, PT 1349-018 Lisboa, Portugal \\ ${ }^{3}$ Centro de Astronomia e Astrofísica da Universidade de Lisboa, Observatório Astronómico de Lisboa, Tapada da Ajuda, 1349-018 Lisboa, Portugal \\ ${ }^{4}$ Leiden Observatory, Leiden University, P.O. Box 9513, NL-2300 RA Leiden, The Netherlands \\ ${ }^{5}$ California Institute of Technology, MC 249-17, 1200 East California Boulevard, Pasadena, CA 91125, USA \\ Received 2015 January 26; accepted 2015 March 27; published 2015 May 27
}

\begin{abstract}
It is well-known that a galaxy's environment has a fundamental influence in shaping its properties. We study the environmental effects on galaxy evolution, with an emphasis on the environment defined as the local number density of galaxies. The density field is estimated with different estimators (weighted adaptive kernel smoothing, 10th and 5th nearest neighbors, Voronoi and Delaunay tessellation) for a $\mathrm{K}_{s}<24$ sample of $\sim 190,000$ galaxies in the COSMOS field at $0.1<z<3.1$. The performance of each estimator is evaluated with extensive simulations. We show that overall there is a good agreement between the estimated density fields using different methods over $\sim 2$ dex in overdensity values. However, our simulations show that adaptive kernel and Voronoi tessellation outperform other methods. Using the Voronoi tessellation method, we assign surface densities to a mass complete sample of quiescent and star-forming galaxies out to $z \sim 3$. We show that at a fixed stellar mass, the median color of quiescent galaxies does not depend on their host environment out to $z \sim 3$. We find that the number and stellar mass density of massive $\left(>10^{11} M_{\odot}\right)$ star-forming galaxies have not significantly changed since $z \sim 3$, regardless of their environment. However, for massive quiescent systems at lower redshifts $(z \lesssim 1.3)$, we find a significant evolution in the number and stellar mass densities in denser environments compared to lower density regions. Our results suggest that the relation between stellar mass and local density is more fundamental than the color-density relation and that environment plays a significant role in quenching star-formation activity in galaxies at $z \lesssim 1$.
\end{abstract}

Key words: galaxies: evolution - large-scale structure of universe - methods: data analysis

Supporting material: machine-readable table

\section{INTRODUCTION}

It is known that the environment in which galaxies reside plays a fundamental role in their evolution. In the local universe, denser environments are dominated by red, passive, early-type galaxies whereas less-dense regions are preferentially populated by blue, star-forming, late-type systems (Dressler 1980; Balogh et al. 2004; Kauffmann et al. 2004; Baldry et al. 2006; Bamford et al. 2009; Peng et al. 2010). These environmental trends still hold at higher redshifts (Capak et al. 2007a; Cooper et al. 2007; Peng et al. 2010; Sobral et al. 2011; Muzzin et al. 2012; Scoville et al. 2013), although they usually tend to weaken with increasing redshift. While it is evident that almost any observable property of a galaxy demonstrates some association with the environment, there is a question that needs to be addressed first. What do we really mean by "environment"?

Recent advances in numerical simulations (Millennium, Springel et al. 2005; Illustris, Vogelsberger et al. 2014) combined with extensive spectroscopic observations of local galaxies (SDSS, York et al. 2000; 2dFGRS, Colless et al. 2001; GAMA, Driver et al. 2011) have revealed that the universe has a web-like pattern (i.e., the "cosmic web"; Bond et al. 1996), containing dark matter, gas, and luminous galaxies. Galaxies in the cosmic web are organized in a network containing dense clusters, sparsely populated voids, planar walls, and thread-like filamentary structures linking overdense regions. Therefore, the most natural approach in defining the environment of a galaxy is to locate it within the cosmic web. However, the complexity and lack of a fully objective method in identifying the major components of the cosmic web often limit the environmental studies of galaxies within the comic web to numerical simulations or large spectroscopic surveys in the local universe.

The conventional method of defining the environment as two extreme regions in the density distribution of galaxies (i.e., galaxy cluster and the general field) does not usually account for the full dynamical range of the density field. For example, there are intermediate environments such as galaxy groups, outskirts of clusters, and filaments that are equally important (Fadda et al. 2008; Porter et al. 2008; Tran et al. 2009; Geach et al. 2011; Coppin et al. 2012; Mahajan et al. 2012; PintosCastro et al. 2013; Darvish et al. 2014). Moreover, the selection of whether galaxies belong to the cluster environment or the general field is somewhat subjective.

The detection of galaxy clusters through their diffuse X-ray emission (Gioia et al. 1990; Ebeling et al. 1998, 2001; Böhringer et al. 2000, 2004; Mehrtens et al. 2012), the red sequence and its variants (Gladders \& Yee 2000, 2005; Goto et al. 2002; Miller et al. 2005; Koester et al. 2007; Hao et al. 2010; Muzzin et al. 2013a), weak gravitational lensing (Dietrich et al. 2007; Gavazzi \& Soucail 2007; Miyazaki et al. 2007) and the Sunyaev-Zel'dovich effect (Marriage et al. 2011; Planck Collaboration et al. 2011; Williamson et al. 2011; Reichardt et al. 2013) adds more assumptions and limitations in the environmental studies. For example, weak lensing, hot Xray emission, and the Sunyaev-Zel'dovich effects are more sensitive to virialized, massive galaxy clusters. The redsequence technique, which relies on observations in only two filters, is very successful and economically efficient in 
detecting a large sample of galaxy clusters that have a tight red sequence of quiescent galaxies. However, this technique assumes the existence of a tight red sequence for clusters, requires modeling the red sequence, and uses only the quiescent systems as a proxy of their host cluster environment. Moreover, the quiescent galaxies become less abundant at higher redshifts, which makes the cluster detection techniques based on the red sequence (or galaxy color) challenging at high redshift. All these methods may produce biased environments, which may lead to misinterpretation of the evolutionary history of galaxies as a function of their host environment.

Another approach in defining the environment is to use the local number density (usually surface density) of galaxies as a proxy to their host region. Among the most common density estimators used in the literature are the nearest neighbor (usually fifth or tenth NN) and count-in-cell (CC) methods. However, although simple to implement and computationally fast, the performance of these methods strongly depends on the depth of the data, the number of neighbors considered in the analysis (in NN method), and the size of the cell (in CC method). A small value of $N$ (a small cell size for CC method) results in a spiky density field that makes it vulnerable to unrealistic density values due to Poisson noise and random clustering of spatially uncorrelated galaxies. A large value of $N$ (a large cell size for CC method) is prone to underestimation of the surface density and oversmoothing the details of galaxy distribution. In addition, for the $\mathrm{NN}$ method, the sum of the area (volume) assigned to each galaxy is not equal to the total area (volume) of any survey and it has also been shown that its integral over all area (volume) diverges.

More importantly, it is still not clear whether the environmental effects depend on the physical scales at which the environment is estimated. For example, Kauffmann et al. (2004), Blanton et al. (2006), and Cucciati et al. (2010) find that the effect of the environment on the star-formation history of galaxies is only effective at small scales $(\lesssim 1 \mathrm{Mpc})$, whereas Balogh et al. (2004) find that the equivalent width of $\mathrm{H} \alpha$ (a measure of the specific star-formation rate (SSFR) in galaxies) is a function of the environment measured on scales of $\sim 1$ and $\sim 5 \mathrm{Mpc}$, independent of each other. Scale-dependent density estimators, such as the NN and CC algorithms, are not able to directly address this issue.

In this work, we perform a comprehensive analysis of the (surface) density field using the weighted versions of the fifth NN, tenth NN, adaptive kernel smoothing, Voronoi tessellation, and Delaunay triangulation methods. This is done using a $\mathrm{K}_{s}$-band selected sample of galaxies in the COSMOS field (Scoville et al. 2007b). The large size of the COSMOS $\left(\sim 2 \mathrm{deg}^{2}\right)$, together with the high accuracy of the photometric redshifts in this field, enable us to delineate the Large Scale Structure with great accuracy out to $z \sim 3$. In this analysis, we use the full photo- $z$ probability distribution function (PDF) of individual galaxies to significantly reduce the projection effect. The performance of each method is checked with extensive realistic and Monte-Carlo simulations. We then apply the algorithm to a mass complete sample of galaxies to investigate the role of the density-based definition of the environment on the rest-frame color evolution of quiescent galaxies, as well as the evolution of the comoving number and mass density of massive systems out to $z \sim 3$.

The format of this paper is as follows. In Section 2, we briefly review the data used to estimate the density field.
Section 3 outlines the algorithm used to determine the density field, followed by the surface density estimation methods in Section 4. Section 5 deals with the simulations used to check the performance of different density estimators. Comparisons between different methods are given in Section 6. In Section 7, we study the color evolution of quiescent galaxies, as well as the number and mass density of massive systems as a function of environment, using a stellar mass complete sample from the COSMOS field. We give a summary of this work in Section 8.

Throughout this work, we assume a flat concordance $\Lambda \mathrm{CDM}$ cosmology with $H_{0}=70 \mathrm{kms}^{-1} \mathrm{Mpc}^{-1}, \Omega_{m}=0.3$, and $\Omega_{\Lambda}=0.7$. All magnitudes are expressed in the AB system and stellar masses are given assuming a Chabrier IMF.

\section{DATA AND SAMPLE SELECTION}

In order to examine different density estimation techniques, we select an area of $\sim 1.8 \mathrm{deg}^{2}$ in the COSMOS field (Capak et al. 2007b; Scoville et al. 2007b). Here, we use the COSMOS UltraVISTA $\mathrm{K}_{s}$-band selected photometric redshift (photo- $z$ ) catalog (McCracken et al. 2012; Ilbert et al. 2013). The $\mathrm{K}_{s^{-}}$ band selection is equivalent to a stellar mass-selected sample and enables us to reliably detect galaxies (especially quiescent systems) at intermediate to high redshifts. This catalog consists of ground- and space-based photometric data in 30 bands, spanning the range UV to mid-IR. Using this extensive data set, accurate photometric redshifts are measured for galaxies out to $z \sim 3$. Stars, active galactic nuclei (AGNs), and X-ray sources were identified and removed from the sample. The final selection criteria used to define the sample are the following.

1. An area of $149.3<\alpha_{2000}<150.8$ and $1.6<\delta_{2000}<2.8$ corresponding to $\sim 1.8 \mathrm{deg}^{2}$. The area is selected to be large enough for the effect of the LSS to be discernible.

2. Redshift within the range $0.05<z<3.2$ to allow photometric redshifts with a high degree of accuracy.

3. Galaxies brighter than $\mathrm{K}_{s}<24$, because the photo- $z$ uncertainties increase significantly at $\mathrm{K}_{s} \gtrsim 24$.

The total sample contains 191,151 galaxies.

\section{DETERMINATION OF THE LARGE SCALE DISTRIBUTION OF GALAXIES}

In any study of the LSS of galaxies, we need the angular position $(\alpha, \delta)$ of the galaxies and a measure of their radial distance (using spectroscopic or photometric redshifts) to estimate the density field. Here, we use the full photo- $z$ PDF of each individual galaxy to significantly reduce the projection effect. In summary, the density field estimation involves the following steps.

1. First, we construct a series of overlapping redshift slices ( $z$-slices). The widths of these slices are obtained by the photo- $z$ accuracy of the underlying sample of galaxies at each redshift.

2. For each $z$-slice, we select a subset of galaxies belonging to that slice. The galaxies in the subset are selected in such a way that the median of their photo- $z$ PDF is located within that $z$-slice. For a given subset, we associate a weight to each galaxy. This weight shows the likelihood of each galaxy belonging to the $z$-slice of our interest. The weight is determined by the photo- $z$ PDF of each galaxy and the boundaries of the $z$-slice. 


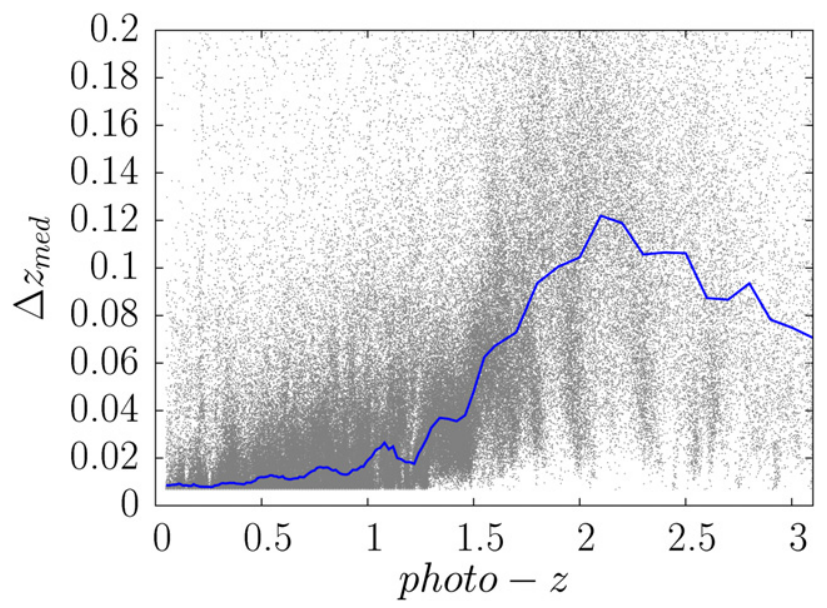

Figure 1. Photo- $z$ uncertainty of galaxies used in this study. The blue solid line shows the median of the photo- $z$ uncertainty $\left(\Delta z_{\text {med }}\right)$ at each redshift. $\Delta z$ for each galaxy is calculated as the (lower and higher) $68 \%$ confidence interval of its photo- $z$ PDF. Note the accuracy of photo- $z$ values. The median of the photo$z$ uncertainty is $\Delta z_{\text {med }} \lesssim 0.01$ out to $z \sim 1$, reaching $\Delta z_{\text {med }} \sim 0.1$ at $z \sim 2$.

3. At each $z$-slice and for its subset of galaxies, we then compute the weighted surface density field using one of the estimation methods described in Section 4.

In the following, we give a detailed description of points 1 and 2 . Using this information, we then apply different density estimators to the data in Section 4.

\subsection{Redshift Slicing}

In order to construct the density field of galaxies, one needs a large, homogeneous, and unbiased sample of galaxies with very accurate redshifts. However, there are serious complications in constructing such samples. First, building a homogeneous sample is difficult. Second, relying on a purely spectroscopic sample would bias the study because it would only concentrate on bright objects and mostly those with emission lines (i.e., star-forming galaxies, which are often less clustered compared to quiescent systems). Spectroscopic samples also do not usually target enough galaxies in dense regions. Third, finding accurate photometric redshifts requires a homogeneous set of multi-waveband photometric data. Thanks to the wealth of the photometric data available in the COSMOS field, we have been able to obtain highly accurate photometric redshifts for a large population of galaxies in this field (Mobasher et al. 2007; Ilbert et al. 2009, 2013). Figure 1 shows the median of photo- $z$ uncertainty $\left(\Delta z_{\text {med }}\right)$ as a function of (photometric) redshift for the sample of galaxies used in this study. $\Delta z$ for each galaxy is calculated as the (lower and higher) $68 \%$ confidence interval of its photo- $z$ PDF. The median of the photo- $z$ uncertainty is $\Delta z_{\text {med }} \lesssim 0.01$ out to $z \sim 1$, reaching $\Delta z_{\text {med }} \sim 0.1$ at $z \sim 2$. Studying the 3D density estimations solely based on the photo- $z$ of galaxies, without taking their photo- $z$ uncertainties into account, may result in an erroneous evaluation of the density field. This is mainly due to the fact that the physical lengths corresponding to even the smallest photo- $z$ uncertainties are larger than the typical sizes of most physical structures, such as galaxy clusters and groups (e.g., at $z \sim 1$, the photo- $z$ uncertainty of $\Delta z \sim 0.01$ is equivalent to the comoving radial length of $\sim 24 \mathrm{Mpc})$. Here, we limit our analysis to the 2D surface density estimations by constructing a series of narrow slices in the redshift space. This is done by defining a series of overlapping redshift slices $(z$ slices). The width of each slice is set by the photometric redshift accuracy of the data at any given redshift. The slices overlap to allow proper contribution from galaxies that reside close to the boundaries of each slice. In practice, we make sure that approximately half of each $z$-slice is trespassed into its adjacent $z$-slices. The width of each $z$-slice $(\delta z)$ at redshift $z$ is defined as twice the median of the photo- $z$ uncertainty $\left(\Delta z_{\text {med }}\right)$ at that redshift:

$$
\delta z=2 \Delta z_{\text {med }}
$$

Figure 1 shows how $\Delta z_{\text {med }}$ changes with redshift. In this study, 133 overlapping $z$-slices were used over the redshift range $0.1<z<3.2$.

Choosing a much narrower $z$-slice results in smaller number statistics and decreased completeness of the underlying sample of galaxies. This subsequently leads to an underestimation of the surface density field. On the other side, broadening the $z$ slice increases the risk of contamination from foreground and background galaxies, as well as a possible overestimation of the density field. Thus, our choice is a compromise between both limits.

\subsection{Photo-z Probability Weights}

To estimate the surface density field, we use the full information of the photo- $z$ PDF for individual galaxies. First, for each $z$-slice, we select a subset of galaxies. Galaxies in each subset are selected in such a way that their median photo- $z$ PDF is located within the boundaries of that $z$-slice. We assign a weight $\left(w_{i}\right)$ to each galaxy in this subset based upon its photo- $z$ PDF. This probability weight is calculated by measuring what percentage of the photo- $z$ PDF of each galaxy is contained within the boundaries of the considered $z$-slice. This weight represents the likelihood that the galaxy in question resides within that $z$-slice. In practice, it is done by intersecting the $z$ slice of our interest with the photo- $z$ PDF of each galaxy in the subset. In order to enhance the computational performance, we assume a Gaussian photo- $z$ PDF for galaxies, except for those that have a second photo- $z$ peak with $P>5 \%$ in their PDF. For the latter, the full photo- $z$ PDF is used in estimating the weights. Eventually, at each redshift and for each $z$-slice, we have a subset of galaxies with associated weights that will later be used as the input for the weighted surface density estimation methods. Figure 2(a) shows the distribution of galaxies for a $z-$ slice centered at $z=0.92$ in the COSMOS. For demonstration, the size of each point is selected to be proportional to the galaxy weight. This subset of galaxies, and their associated weights, is used to estimate the weighted surface density field at $z=0.92$.

We note that the weight introduced in this section can be generalized to other galaxy properties, such as stellar mass, luminosity, and color, depending on the astrophysics of our problem.

\section{SURFACE DENSITY ESTIMATION: THE METHODS}

Using the information obtained in Section 3, we now construct the density field for galaxies in the COSMOS, using weighted versions of four independent methods: adaptive kernel, NN, Voronoi tessellation, and Delaunay triangulation 
(a)

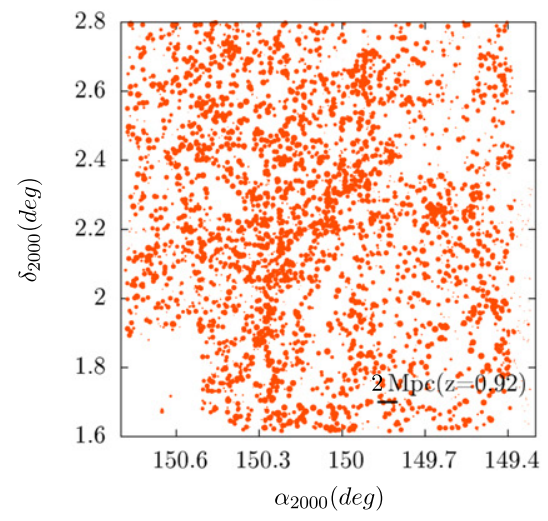

(c)

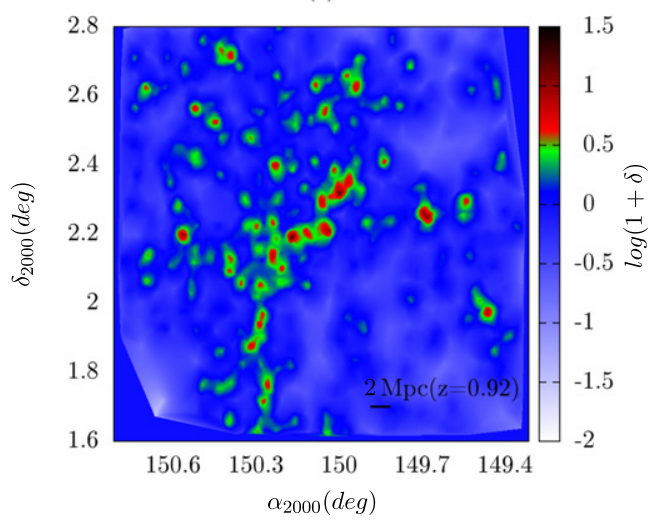

(e)

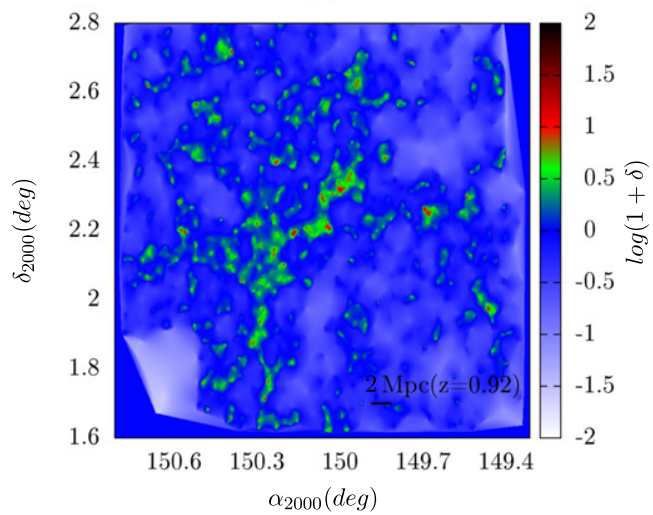

(b)

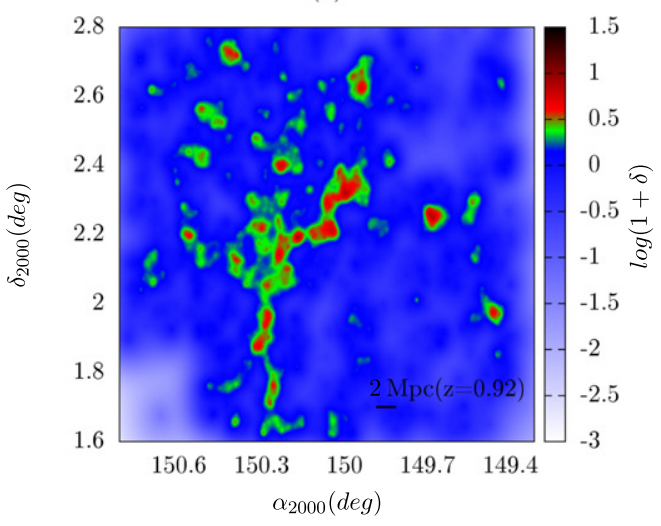

(d)

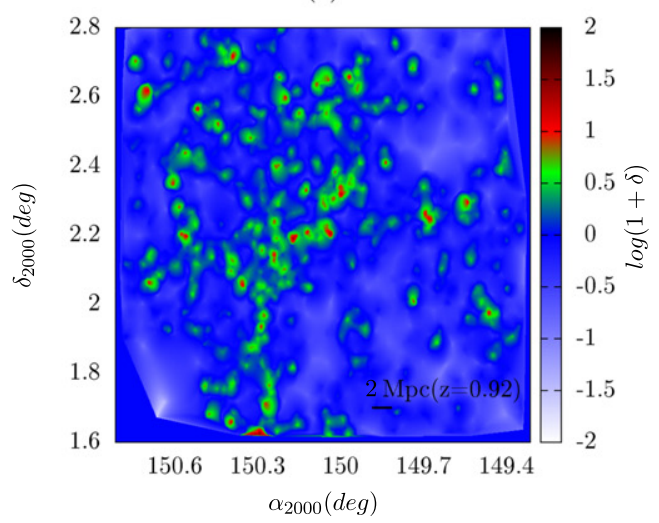

(f)

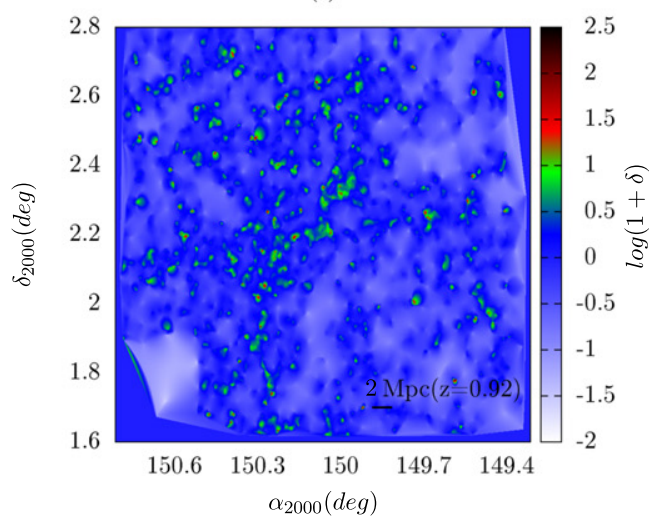

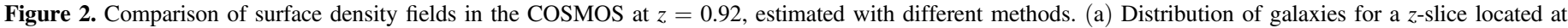

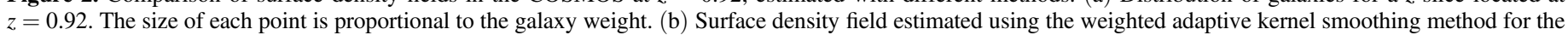

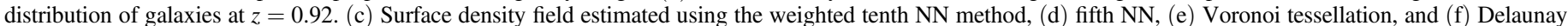
triangulation methods.

(Sections 4.1-4.4). We then evaluate their performance with simulations in Section 5 and compare them with each other in Section 6.

\subsection{Weighted Adaptive Kernel Estimator}

In this method, we use an iterative procedure to compute the surface density field (Darvish et al. 2014). First, we estimate the surface density associated with the $i$ th galaxy in a given $z$ slice, $\hat{\Sigma}_{i}$, by summing over all the weighted fixed kernels placed on the positions of galaxies, $j$, where $i \neq j$ :

$$
\hat{\Sigma}_{i}=\frac{1}{\sum_{\substack{j=1 \\ j \neq i}}^{N} w_{j}} \sum_{\substack{j=1 \\ j \neq i}}^{N} w_{j} K\left(\boldsymbol{r}_{i}, \boldsymbol{r}_{j}, h\right),
$$

where $N$ is the number of galaxies in the subset, $K\left(\boldsymbol{r}_{i}, \boldsymbol{r}_{j}, h\right)$ is the fixed kernel, $\boldsymbol{r}_{i}$ is the position of the galaxy for which the initial estimate of surface density is measured, and $\boldsymbol{r}_{j}$ is the position of the rest of the galaxies. The width of the kernel is 
expressed by the parameter, $h$, which is a proxy for the degree of smoothing. For the first estimate of the density, this is taken to be fixed. For the kernel smoothing function, we use a 2D symmetric Gaussian defined as:

$$
K\left(\boldsymbol{r}_{i}, \boldsymbol{r}_{j}, h\right)=\frac{1}{2 \pi h^{2}} e^{-\frac{\left|\boldsymbol{r}_{i}-\boldsymbol{r}_{j}\right|^{2}}{2 h^{2}}}
$$

A large kernel width $(h)$ results in oversmoothing of the density field, which tends to wash out real features, whereas a small value tends to break up regions into smaller uncorrelated substructures. Here, we use a fixed physical length of $h=0.5 \mathrm{Mpc}$, which corresponds to the typical value of $R_{200}$ for X-ray clusters and groups in the COSMOS field (Finoguenov et al. 2007; George et al. 2011). However, a constant value of $h$ for the whole field underestimates the surface density in crowded regions while overestimating in sparsely populated areas. To overcome this problem, we introduce the adaptive smoothing width, $h_{i}$, which is a measure of the local surface density associated with each galaxy, $\hat{\Sigma}_{i}$. This is defined as $h_{i}=h \times \lambda_{i}$, where $\lambda_{i}$ is a parameter that is inversely proportional to the square root of the surface density associated with the $i$ th galaxy, at the position of that galaxy (Silverman 1986):

$$
\lambda_{i}=\left(G / \hat{\Sigma}\left(\boldsymbol{r}_{1}\right)\right)^{0.5}
$$

where $G$ is the geometric mean of all the $\hat{\Sigma}\left(\boldsymbol{r}_{i}\right)$ values. Having the adaptive kernel, we now calculate the surface density field, $\Sigma(\boldsymbol{r})$, at each location on a fine $2 \mathrm{D}$ grid, $\boldsymbol{r}=(x, y)$ as:

$$
\Sigma(\boldsymbol{r})=\frac{1}{\sum_{i=1}^{N} w_{i}} \sum_{i=1}^{N} w_{i} K\left(\boldsymbol{r}, \boldsymbol{r}_{i}, h_{i}\right) .
$$

The surface density field is evaluated on a fine grid with a grid size (resolution) of $50 \mathrm{Kpc}$ at each redshift. We note that the surface density field estimated through this method is almost independent of the type of the kernel function. We examined several other standard kernels, including exponentially decaying, Epanechnikov $\left(K(r) \propto\left(1-r^{2}\right)\right)$, and cosine arch $(K$ $\left.(r) \propto \cos \left(\frac{\pi}{2} r\right)\right)$, and did not find any significant difference in the final results. Throughout this work, we define overdensity as:

$$
1+\delta=\frac{\Sigma}{\Sigma_{\text {median }}},
$$

where $\Sigma_{\text {median }}$ is the median of the surface density field at each redshift. Figure 2(b) shows an example of the surface density field for a $z$-slice in the COSMOS field centered at $z=0.92$, using the weighted adaptive kernel density estimator. Note the wide range of overdensities and the variety of environments, including dense regions linked together through thread-like filamentary structures.

In Table 1 we provided an example of the overdensity values estimated with the adaptive kernel smoothing method for our sample galaxies (Section 2). $\mathrm{K}_{s}$ magnitudes and photo- $z$ estimates are extracted from publicly available catalogs of McCracken et al. (2012) and Ilbert et al. (2013), respectively. We recommend using galaxies that are far from the edge of the
Table 1

Overdensity Values Evaluated Using the Adaptive Kernel Estimator for Sample Galaxies Selected in Section 2

\begin{tabular}{cccccc}
\hline \hline ID & $\begin{array}{c}\alpha_{2000} \\
\text { deg }\end{array}$ & $\begin{array}{c}\delta_{2000} \\
\text { deg }\end{array}$ & Photo- $z$ & $\begin{array}{c}\mathrm{K}_{s} \\
\mathrm{mag}\end{array}$ & $\log \left(\Sigma / \Sigma_{\text {median }}\right)_{\text {kernel }}$ \\
\hline 1 & 150.02025 & 1.67951 & 0.0532 & 22.69 & -0.5811 \\
2 & 150.39144 & 1.68383 & 0.0506 & 23.30 & -0.6667 \\
3 & 149.58068 & 1.68829 & 0.0512 & 23.71 & -0.4306 \\
\hline
\end{tabular}

(This table is available in its entirety in machine-readable form.)

field and/or the masked regions. A catalog of overdensity values with other density estimation methods is also available upon request.

\subsection{Weighted $k-N N$ Estimator}

In a regular (non-weighted) $k$-NN method, the inverse of the area containing the $k$ th NNs to each galaxy is used as a proxy for local surface density at the position of that galaxy. Because the galaxies in our sample are weighted, we incorporate the role of weights in the density estimation. The surface density at the position of the $i$ th galaxy, $\Sigma\left(\boldsymbol{r}_{i}\right)$, is estimated as:

$$
\Sigma\left(\boldsymbol{r}_{i}\right)=\frac{\sum_{j=1}^{k} j w_{i j}}{\pi \sum_{j=1}^{k} w_{i j} d_{i j}^{2}},
$$

where $\boldsymbol{r}_{i}$ is the position of $i$ th galaxy, $w_{i j}$ is the photo- $z$ probability weight (Section 3.2) associated with the $j$ th NN to the galaxy located at $\boldsymbol{r}_{i}$, and $d_{i j}$ is the distance between them (the distance between the galaxy positioned at $\boldsymbol{r}_{i}$ and its $j$ th neighbor). Using the distance to the fifth $(k=5)$ or tenth $(k=10) \mathrm{NN}$ is very common in the literature (see for example, Cooper et al. 2005; Sobral et al. 2011). In this work, we use the weighted versions of both the fifth $\left(\mathrm{NN}_{5}\right)$ and tenth $\left(\mathrm{NN}_{10}\right) \mathrm{NN}$ estimators. Figures 2(c) and (d) show examples of the surface density field at $z=0.92$, estimated using $\mathrm{NN}_{10}$ and $\mathrm{NN}_{5}$, respectively. The $\mathrm{NN}_{5}$ method gives a larger density value in very dense regions compared to $\mathrm{NN}_{10}$, as expected. The overall traces of $\mathrm{NN}_{5}$ are seen in the $\mathrm{NN}_{5}$ plot. However, the $\mathrm{NN}_{5}-$ based density field looks spikier and clumpier due to the smaller physical lengths that the $\mathrm{NN}_{5}$ method spans.

\subsection{Weighted Voronoi Tessellation Estimator}

In a simple Voronoi tessellation method, we divide the $2 \mathrm{D} z-$ slice plane into a number of regions (Voronoi cells) assigned to each galaxy located at $\boldsymbol{r}_{i}$. The Voronoi cell of each galaxy is defined as all points in the $z$-slice plane that are closer to that galaxy than to any other galaxy. It is acquired from the intersection of half-planes. Based on this definition, in more crowded, denser regions the Voronoi cells of galaxies incline toward smaller values. Therefore, we can use the inverse of the area of the Voronoi cell of each galaxy as a measure of the local density at the position of that galaxy (Ebeling \& Wiedenmann 1993; Bernardeau \& van de Weygaert 1996). This is given by:

$$
\Sigma\left(\boldsymbol{r}_{i}\right)=\frac{1}{A_{i}},
$$


where $\Sigma\left(\boldsymbol{r}_{i}\right)$ is the surface density at the position of the $i$ th galaxy and $A_{i}$ is the area of its Voronoi cell.

In order to assimilate the role of galaxy weights, we tried two different approaches. In the first approach, we modified the metric used for the definition of distance between the points in the $z$-slice plane and galaxies. This was done by dividing the Euclidean metric by the weight of each galaxy. Implementing this approach to the data was not successful and resulted in unrealistically high-density values for the majority of galaxies (with small weights) located in sparsely populated regions. Thus, we alternatively use a Monte-Carlo acceptance-rejection process in order to take the role of weights into consideration. The steps are as follows.

1. For each galaxy with its corresponding weight $w_{i}$, we generate a random number $R_{i}$ between the minimum and maximum weight values in each $z$-slice.

2. If $w_{i}>R_{i}$, we accept the galaxy (with its associated weight $w_{i}$ ) in the density estimation procedure.

3. We estimate the surface density for the accepted galaxies using a simple Voronoi tessellation method. This surface density is evaluated at the position of accepted galaxies only.

4. We interpolate ${ }^{6}$ the estimated densities into the points of a grid $(\boldsymbol{r})$ (the grid resolution is $50 \mathrm{Kpc}$ at each redshift) constructed on the $z$-slice plane. This gives us a MonteCarlo estimated surface density field $\tilde{\Sigma}(\boldsymbol{r})$.

5. We repeat this procedure $N$ times and take the mean of all the Monte-Carlo density fields as the actual density field $\Sigma(\boldsymbol{r})$ :

$$
\Sigma(\boldsymbol{r})=\frac{1}{N} \sum_{m=1}^{N} \tilde{\Sigma}_{m}(\boldsymbol{r}) .
$$

Finally, the local surface density of each galaxy is then estimated as that of its closest point in the grid constructed over the $z$-slice plane. To save computational time, we use $N=10$ in step 5. Figure 2(e) shows an example of the surface density field at $z=0.92$, estimated with the weighted Voronoi tessellation algorithm. Note the large dynamical range of overdensities. Unlike the NN, Voronoi tessellation is scaleindependent and is able to span a wide range of physical lengths. In addition, it does not make any assumptions about the geometry and morphology of the structures in the density field. This characteristic makes it superior to adaptive kernel and NN methods.

\subsection{Weighted Delaunay Triangulation Estimator}

This method relies on segmenting the $z$-slice plane into triangles whose vertices are defined by the position of galaxies in the $z$-slice plane. For each triangle, these three vertices (position of galaxies) are selected such that their circumcircle does not encompass any other galaxy. In this method, each galaxy is eventually surrounded by a series of neighboring triangles whose overall area tend to be smaller in denser regions (Schaap \& van de Weygaert 2000; Platen et al. 2011). For the $i$ th galaxy surrounded by $m$ neighboring triangles, the estimated surface density is expressed as the inverse of the sum

\footnotetext{
6 We use the natural neighbor interpolation developed by Sibson.
}

of the areas of its neighboring triangles:

$$
\Sigma\left(\boldsymbol{r}_{i}\right)=\frac{1}{\sum_{n=1}^{m} a_{n}},
$$

where $\Sigma\left(\boldsymbol{r}_{i}\right)$ is the surface density at the position of the $i$ th galaxy and $a_{n}$ is the area of the $n$th triangle neighboring the $i$ th galaxy.

In order to take the weight of galaxies into account, we utilize a Monte-Carlo acceptance-rejection approach explained in Section 4.3. Figure 2(f) shows an example of the surface density field at $z=0.92$, estimated with the weighted Delaunay method. When compared with other estimation methods, this method overestimates the densities in very dense regions and the resulting density field is clumpier and contains much more substructures.

\section{SIMULATIONS}

In order to evaluate the performance of each surface density estimation method, we run two sets of simulations. The details are given in the following subsections.

\subsection{Simulation 1}

In the first set of simulations, we apply the surface density estimators to a sample of galaxies that was randomly drawn from some previously known surface density profiles (Scoville et al. 2007a). Since the simulated surface density values are known, we can directly compare them with the estimated surface densities predicted by each of our density estimators. Here, we make 30 different simulated structures. These structures are placed on an area similar to that of the COSMOS field. The simulated structures and the field contain 5000 galaxies at $z=1$, similar to the total number of observed galaxies at that redshift in the COSMOS field. For simplicity, we assume that all have the same weight $(w=1) .2222$ of these galaxies are randomly distributed on an area covering $1.5 \times 1.2 \mathrm{deg}^{2}$. The rest are drawn randomly from a series of Gaussian profiles. These Gaussian structures have a variety of sizes $(0.1-2 \mathrm{Mpc})$ and the number of galaxies in these structures ranges from 3 to 300 . The properties of these structures are shown in Table 2 . We apply the density field estimation methods explained in Section 4 to the simulated galaxies to explore how well they can predict the input density field. Figure 3 shows the density field of the simulated structures, the distribution of galaxies randomly drawn from the structures, and the predicted density fields using different estimation methods. All these methods perform relatively well when compared to the expected density field. We stress that some of the difference between the expected and predicted density fields is caused by the shot noise, which becomes specifically important when the number of galaxies in simulated structures is small and the width of the profile is large. In order to quantify the performance of different density estimation methods, we define the mean squared error (MSE) as:

$$
\operatorname{MSE}=\frac{1}{N} \sum_{i=1}^{N}\left(\Delta_{i}^{\mathrm{sim}}-\Delta_{i}\right)^{2},
$$

where $N$ is the total number of generated galaxies, $\Delta_{i}^{\text {sim }}=\log$ $\left(1+\delta_{i}^{\text {sim }}\right)$ is the logarithm of the overdensity of the $i$ th galaxy in the simulation, and $\Delta_{i}$ is a similar quantity, predicted by one 
Table 2

Properties of Simulated Structures

\begin{tabular}{|c|c|c|c|c|}
\hline Structure & $\begin{array}{c}\alpha_{2000} \\
\operatorname{deg}\end{array}$ & $\begin{array}{c}\delta_{2000} \\
\text { deg }\end{array}$ & $\begin{array}{c}\sigma \\
\mathrm{Mpc}\end{array}$ & $N$ \\
\hline 1 & 149.5 & 1.8 & 0.1 & 300 \\
\hline 2 & 149.7 & 1.8 & 0.2 & 300 \\
\hline 3 & 149.9 & 1.8 & 0.5 & 300 \\
\hline 4 & 150.1 & 1.8 & 1.0 & 300 \\
\hline 5 & 150.3 & 1.8 & 1.5 & 300 \\
\hline 6 & 150.5 & 1.8 & 2.0 & 300 \\
\hline 7 & 149.5 & 2.0 & 0.1 & 100 \\
\hline 8 & 149.7 & 2.0 & 0.2 & 100 \\
\hline 9 & 149.9 & 2.0 & 0.5 & 100 \\
\hline 10 & 150.1 & 2.0 & 1.0 & 100 \\
\hline 11 & 150.3 & 2.0 & 1.5 & 100 \\
\hline 12 & 150.5 & 2.0 & 2.0 & 100 \\
\hline 13 & 149.5 & 2.2 & 0.1 & 50 \\
\hline 14 & 149.7 & 2.2 & 0.2 & 50 \\
\hline 15 & 149.9 & 2.2 & 0.5 & 50 \\
\hline 16 & 150.1 & 2.2 & 1.0 & 50 \\
\hline 17 & 150.3 & 2.2 & 1.5 & 50 \\
\hline 18 & 150.5 & 2.2 & 2.0 & 50 \\
\hline 19 & 149.5 & 2.4 & 0.1 & 10 \\
\hline 20 & 149.7 & 2.4 & 0.2 & 10 \\
\hline 21 & 149.9 & 2.4 & 0.5 & 10 \\
\hline 22 & 150.1 & 2.4 & 1.0 & 10 \\
\hline 23 & 150.3 & 2.4 & 1.5 & 10 \\
\hline 24 & 150.5 & 2.4 & 2.0 & 10 \\
\hline 25 & 149.5 & 2.6 & 0.1 & 3 \\
\hline 26 & 149.7 & 2.6 & 0.2 & 3 \\
\hline 27 & 149.9 & 2.6 & 0.5 & 3 \\
\hline 28 & 150.1 & 2.6 & 1.0 & 3 \\
\hline 29 & 150.3 & 2.6 & 1.5 & 3 \\
\hline 30 & 150.5 & 2.6 & 2.0 & 3 \\
\hline
\end{tabular}

Note. The structures have a Gaussian profile with a variety of sizes and number of galaxies.

of the density estimator methods. A smaller value of MSE shows a higher similarity between the simulation and predicted density fields, and is a measure of the performance of the estimation methods in predicting the true value of the density field. The MSE values are tabulated in Table 3. It is clear that the adaptive kernel, Voronoi tessellation, and $\mathrm{NN}_{10}$ methods outperform the $\mathrm{NN}_{5}$ and Delaunay triangulation algorithms.

We note that due to the catastrophic failure in estimating the photometric redshifts, the actual discrepancy between the simulated and predicted density fields can be higher. However, the catastrophic failure fraction (defined as the fraction of galaxies that satisfy $\left.\left|z_{\text {phot }}-z_{\text {spec }}\right| /\left(1+z_{\text {spec }}\right)>0.15\right)$ for $\mathrm{K}_{s}<24$ galaxies in the COSMOS field) is relatively small and estimated to be $<1 \%$ for bright sources, reaching $\lesssim 10 \%$ for fainter galaxies (Ilbert et al. 2013). We redo the simulation taking into account the catastrophic failure and estimate the new MSE statistics. We assume that $10 \%$ of the simulated galaxies are affected by the catastrophic failure (i.e., they do not actually belong to the simulated $z$-slice) and they are randomly distributed over the area containing the simulated structures (random distribution is a fair assumption because the catastrophic failure mostly affects fainter galaxies, which tend to be less clustered). This means that the actual background for the overdensity map of the simulated structures should have a smaller value. With the new simulation, all density field estimators perform almost equally worse with catastrophic failure (as predicted). The MSE statistics are increased by $\sim 0.01-0.02(\sim 3 \%-6 \%)$ for all density estimators. In this work, we do not correct for the catastrophic failure, but we highlight that it has a small effect and that the trends are not affected much by it.

\subsection{Simulation 2}

In the previous simulations, although the "true" density values are known (which makes the comparison with the predicted densities feasible), the complex geometry of the real astronomical density fields and their diverse dynamical range are not fully considered; the simulated density profiles have simple mathematical shapes, whereas the real astronomical data consist of an irregular web of filaments, voids, walls, and clusters with a variety of physical scales and geometries. Furthermore, the previous simulation greatly suffers from the shot noise, especially when the number of randomly drawn galaxies is small and/or the known density profiles have broad, close-to-the-field shapes. In order to consider the complexity of the cosmic web in the analysis, one can use the numerical simulations that produce mock galaxy distributions, which resemble the real density maps. Since the "true" density field is unknown in these quasi-real simulations, we should rely on an estimator to have an initial guess of the density field. In the second set of simulations, we make a sample of galaxies that resemble the true distribution of galaxies in the COSMOS. This is performed using a Monte-Carlo acceptancerejection approach, which is a method similar to the one explained in Section 4.4, taking the following steps.

1. For each $z$-slice, we estimate the surface density field for the real data using one of the density estimators explained in Section 4. For simplicity, we assume that all galaxies in the $z$-slice have the same weight $(w=1)$. The estimated density field gives us a first order approximation of the shape of the true density field.

2. For each $z$-slice, we randomly select a position, $(x$, $y)_{\text {random, }}$ in the area covering the data and assign a random number, $\Sigma_{\text {random }}$, between the minimum and maximum density values in that $z$-slice to the point $(x$, $y)_{\text {random. We also report the density value at this random }}$ position from step 1 and call it $\hat{\Sigma}$.

3. If $\hat{\Sigma}>\Sigma_{\text {random }}$, we accept the point $(x, y)_{\text {random }}$ as one of the points in the simulated data set and consider $\hat{\Sigma}$ as its true density.

4. For each $z$-slice, we repeat steps 1-3 until we have a simulated data set with the same number of galaxies as the actual data set.

Given the above steps, for each $z$-slice we now have a sample of simulated galaxies with known surface densities. The distribution of these galaxies resembles the actual data in the COSMOS. We apply our surface density estimation methods to the simulated data with known densities and compare their expected and predicted densities. Although we now consider the complexity of the real galaxy distribution, these simulations rely on an initial estimator to determine the rough shape of the density field (i.e., step 1). We therefore use the adaptive kernel density estimator in order to perform the first step of the simulation. ${ }^{7}$ Table 4 shows the performance of different surface density estimators when

\footnotetext{
7 This results in a bias in favor of the performance of the adaptive kernel density estimator when we compare it with the simulations. However, we used other estimators as well to initiate the simulation and found that the adaptive kernel estimator has an overall relatively good performance in all these simulations.
} 
(a)

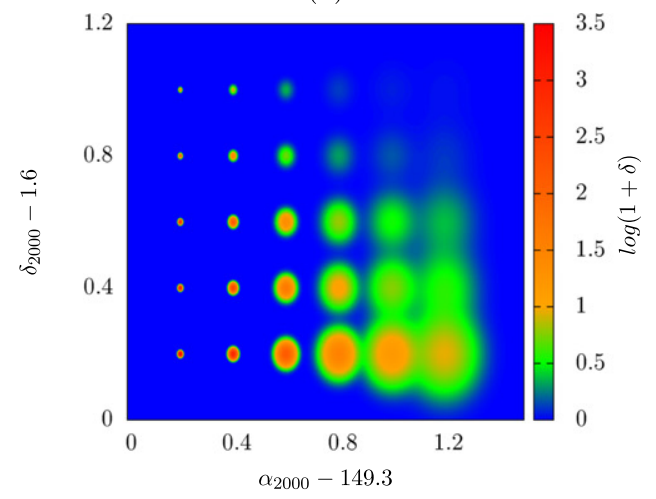

(c)

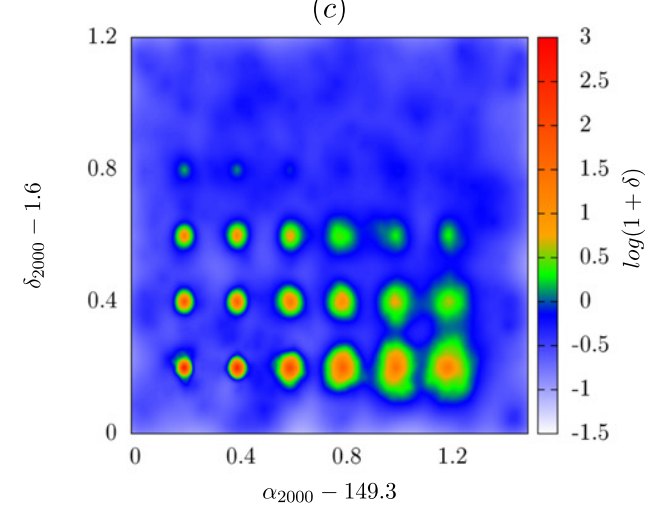

$(e)$

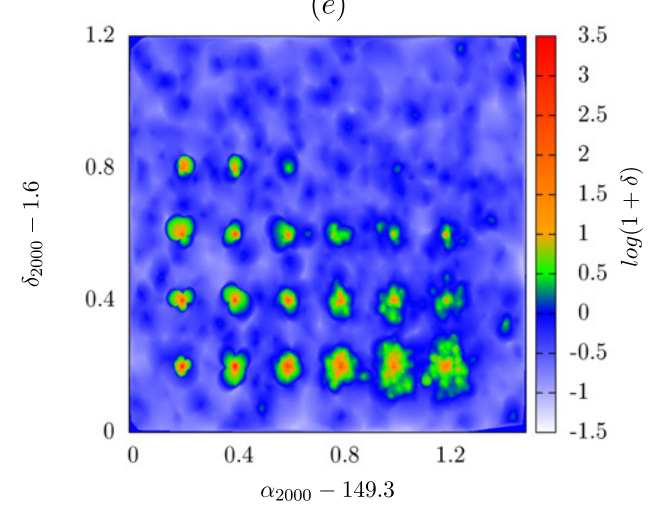

$(g)$

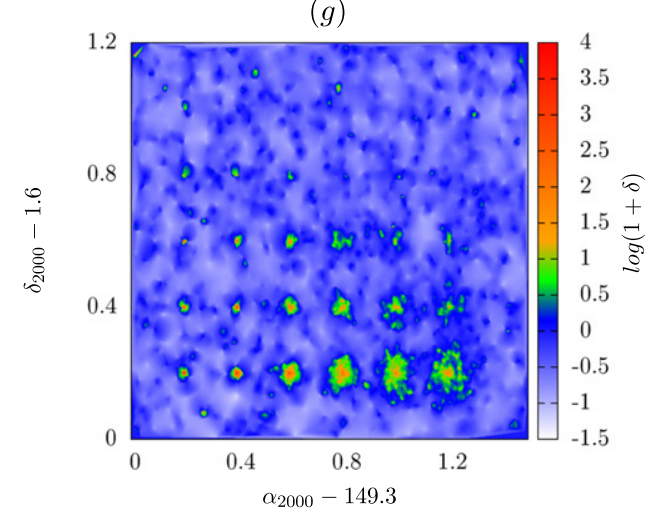

(b)

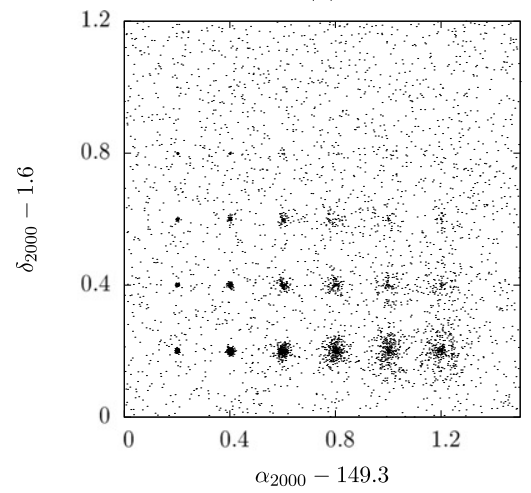

$(d)$

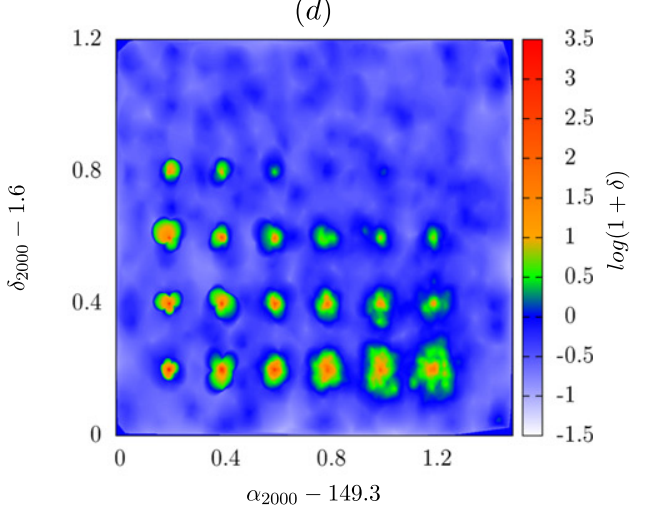

$(f)$

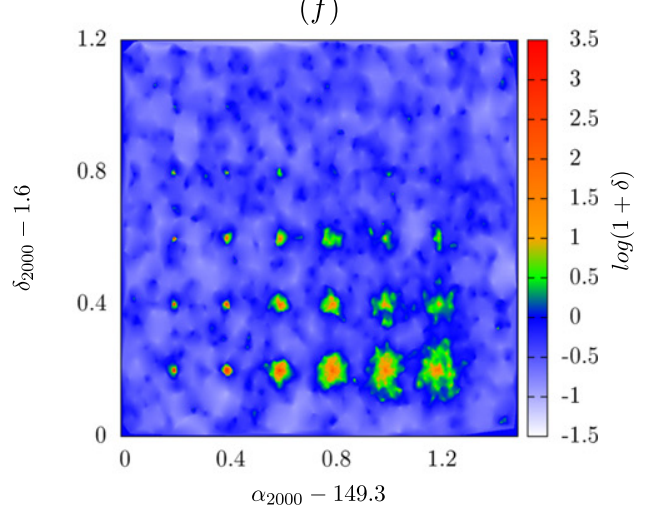

Figure 3. (a) Overdensity map for simulated structures. Here, we make 30 different simulated structures placed on a constant background. They are assumed to be located at $z=1$ and cover an area similar to that of the COSMOS field. The structures have Gaussian profiles with a variety of sizes $(0.1-2 \mathrm{Mpc})$ and the number of galaxies in these structures ranges from 3 to 300. (b) Distribution of galaxies randomly drawn from the structures. The simulated structures and the field contain 5000 galaxies. Almost half of them (2778) belong to the structures while the rest are randomly distributed on an area covering $1.5 \times 1.2 \mathrm{deg}^{2}$, which serve like the field. (c) Predicted overdensity maps using the adaptive kernel, (d) tenth NN, (e) fifth NN, (f) Voronoi tessellation, and (g) Delaunay triangulation. All these methods perform relatively well when compared to the expected density field, with the adaptive kernel and Voronoi tessellation performing relatively better than other estimators. 
Table 3

Comparison Between the Simulated Density Field (Simulation 1) and the One Predicted by Different Estimators

\begin{tabular}{cccccc}
\hline \hline & Kernel & $\mathrm{NN}_{10}$ & $\mathrm{NN}_{5}$ & Voronoi & Delaunay \\
\hline MSE & 0.26 & 0.28 & 0.30 & 0.27 & 0.34 \\
\hline
\end{tabular}

Note. We use the MSE measure for comparison. A smaller value of MSE indicates a better performance.

Table 4

Comparison Between the Simulated Density Field (Simulation 2) and the One Predicted by Different Estimators

\begin{tabular}{cccccc}
\hline \hline & Kernel & $\mathrm{NN}_{10}$ & $\mathrm{NN}_{5}$ & Voronoi & Delaunay \\
\hline MSE & 0.003 & 0.035 & 0.062 & 0.025 & 0.158 \\
\hline
\end{tabular}

Note. We use the MSE measure for comparison. A smaller value of MSE indicates a better performance.

compared with the new set of simulations. Here, we use the MSE measure introduced in Section 5.1 to compare the results. According to the new simulations and the results of the MSE statistics, the adaptive kernel, Voronoi tessellation, and $\mathrm{NN}_{10}$ methods perform better in estimating the simulated density field. In both sets of simulations, the $\mathrm{NN}_{5}$ and (especially) the Delaunay tessellation algorithms seem to fall behind compared to other estimators.

\section{COMPARISON}

Using two sets of simulations, we showed that all density estimation methods perform relatively well in estimating the density field. However, the adaptive kernel, Voronoi tessellation, and $\mathrm{NN}_{10}$ outperformed the others. In this section, we compare different estimation methods regardless of their performance. Due to the edge effect, the surface density of the galaxies located near the edge of the survey and masked areas are unrealistically underestimated. In order to take this into account, we limit the comparison to galaxies that are more than $1 \mathrm{Mpc}$ (physical) away from the edge of survey and large masked areas. Figure 4 shows a comparison between different estimation methods using 176893 galaxies at $0.1<z<3.2$. There is a relatively good agreement between different estimation methods over $\sim 2$ orders of magnitude in overdensity values. However, when we compare the Delaunay triangulation method with the rest, it overestimates the density values in dense regions while underestimating the density in sparsely populated regions (field) when compared with adaptive kernel and $\mathrm{NN}_{10}$ methods.

Each surface density estimator method has its own advantages and disadvantages, as explored below.

1. Adaptive Kernel: we smooth the density field with a suitable kernel function, so this method is less affected by the shot noise and possible random clustering of foreground and background sources. Calculation of the global kernel width $(h)$ is motivated based on some physical scales (e.g., the typical size of galaxy clusters and groups), which makes it suitable for practical observational situations. We can easily add weights to the estimator by multiplying the kernel function with a proper weight. This method conserves the total number of galaxies (i.e., the integral of the surface density field over the whole area yields the total number of galaxies). However, the selection of the appropriate kernel size is a serious problem, because a small kernel size tends to overestimate the sparsely populated regions in the density field whereas a large kernel size washes out real features. This is partly overcome by adaptively changing the kernel width, but at the expense of increasing the computational time by adding an extra step to initially estimate the density field at the position of galaxies. Because we estimate the surface density at the positions of a grid, an intermediate interpolation is required to assign the density values from the grid points to the position of the galaxies. It is also computationally expensive in its adaptive format because it requires extra steps to determine the local adaptive kernel width.

2. $k$-NN: it is by far the easiest method to implement and the fastest to perform computationally. It estimates the surface density directly at the position of galaxies, which does not require any interpolation. Adding weights to this method is readily done. However, its performance strongly depends on the number of neighbors considered in the analysis $(k)$. A small value of $k$ results in a spiky density field that makes it vulnerable to unrealistic density values due to Poisson noise and random clustering of spatially uncorrelated galaxies. A large value of $k$ is prone to underestimation of the surface density and oversmoothing the details of galaxy distribution. The sum of the area assigned to each galaxy is not equal to the total area of survey. It has also been shown that its integral over all areas diverges.

3. Voronoi Tessellation: this method covers a wide dynamical range in densities and is able to estimate densities in a broad range, from the dense core of clusters to sparse regions devoid of galaxies. It is a nonparametric and scale-independent method that makes no prior assumption about the shape of the density field. We directly evaluate the surface density at the position of the galaxies. However, we cannot assign closed Voronoi areas to galaxies near the edge of the field. Adding weights to this method is not straightforward. Here, we used a Monte-Carlo method to take the role of weights into consideration. However, this comes at the expense of a computationally expensive process, by making several Monte-Carlo samples. Apart from the computational time, it is a robust estimator.

4. Delaunay Tessellation: in terms of advantages and disadvantages, it is very similar to the Voronoi tessellation method. However, we can assign a delaunay area to all galaxies in the sample, even those that are located at the edge of the field. Also in this method, the total area assigned to galaxies surpasses the area of the survey. Despite its similarity to the Voronoi tessellation, this method does not perform well compared to other estimation methods (at least for the COSMOS field).

Due to the good performance of the Voronoi tessellation method, its large dynamical range, scale independence, and no prior assumption it makes about the morphology of the structures, we use it for the scientific analysis in the next sections. Figure 5 shows the projected overdensity map in the whole COSMOS field at $0.1<z<3.2$ using the weighted Voronoi tessellation method. In constructing this, we stack the overdensity maps from all the $z$-slices between $z=0.1$ and 

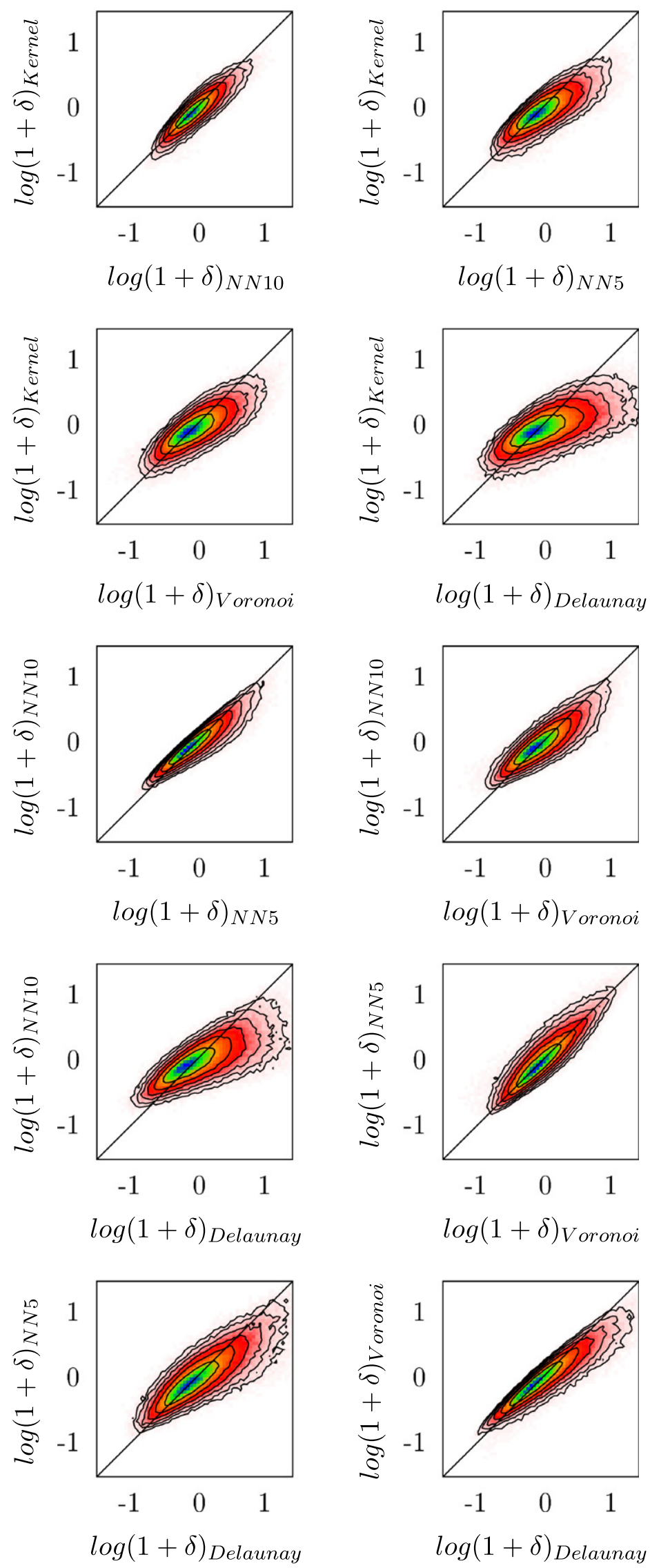

Figure 4. Comparison between different estimation methods using 176,893 galaxies at $0.1<z<3.2$. In order to minimize the edge effect, we limit the comparison to galaxies that are $1 \mathrm{Mpc}$ away from the edge of the field and large masked regions. There is a good agreement between different estimation methods over $\sim 2$ orders of magnitude in overdensity values. However, when we compare the Delaunay triangulation method with the rest, it overestimates the density values in dense regions while underestimating the density in sparsely populated areas. 


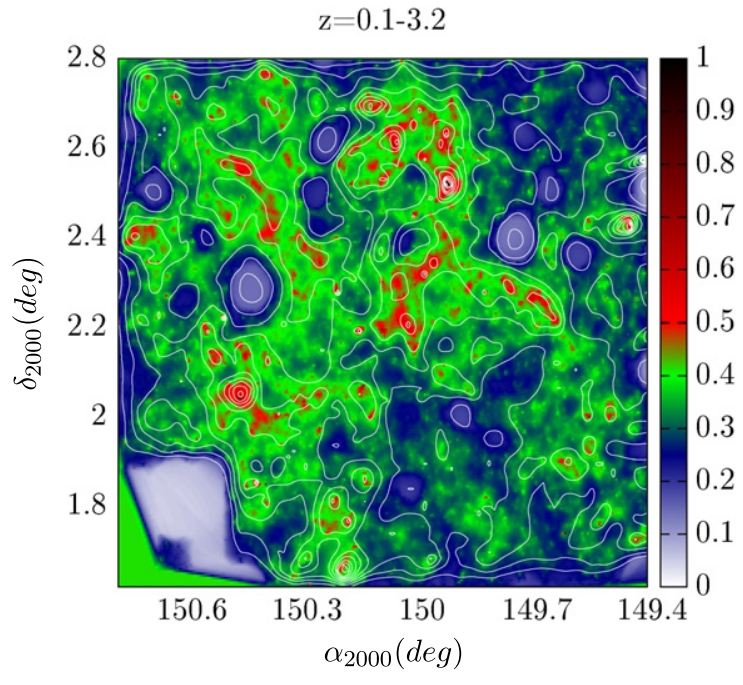

Figure 5. Projected overdensity map in the whole COSMOS field at $0.1<z<3.2$, using the weighted Voronoi tessellation method. In constructing this, the overdensity maps from all the $z$-slices between $z=0.1$ and $z=3.2$ are stacked and normalized to the peak. We compare it with the stacked overdensity map from Voronoi-based algorithm of Scoville et al. (2013). The contours used to demonstrate the map from Scoville et al. (2013) are at levels $0.2-1$ with 0.05 spacing between them. There is a very good agreement between Scoville et al. (2013) and our work. We also find a relatively good agreement between the denser regions in our work and the position of X-ray clusters/groups (Finoguenov et al. 2007; George et al. 2011), as well as the projected mass map from the weak lensing analysis of Massey et al. (2007) in the COSMOS.

$z=3.2$, and normalize it to the peak of stacked overdensities. We compare it with the stacked overdensity map from Voronoi-based algorithm of Scoville et al. (2013). The contours used to demonstrate the map from Scoville et al. (2013) are at levels $0.2-1$ with 0.05 spacing between them. In terms of sample selection, our work is similar to that of Scoville et al. (2013). We see very good agreement between Scoville et al. (2013) and our work. We also find a relatively good agreement between the denser regions in our work and the position of the X-ray clusters and groups (Finoguenov et al. 2007; George et al. 2011). When compared with the projected mass map from the weak lensing analysis of Massey et al. (2007) in the COSMOS field, our projected map agrees with that of Massey et al. (2007). We are able to recover all the massive structures in the weak lensing map. Our estimated density field is also consistent quantitatively with the density field of Kovač et al. (2010), who used zCOSMOS spectroscopic data out to $z \sim 1$, the optical galaxy groups of Knobel et al. $(2009,2012)$ using the $z$ COSMOS data set, and the protocluster candidates of Chiang et al. (2014) at $1.6<z<3.1$ in the COSMOS field. In the following sections, we use the weighted Voronoi tessellation method to study the dependence of the observable parameters on the environment.

\section{DEPENDENCE OF THE PHYSICAL PROPERTIES OF GALAXIES ON THE ENVIRONMENT}

Using the surface density field constructed in the COSMOS field, we now study the dependence of the observable parameters of galaxies on their local density. We first need to define a mass complete sample of galaxies at different redshifts. In the following sections, we select the sample and then use it to investigate the role of environment on the properties of the galaxies.

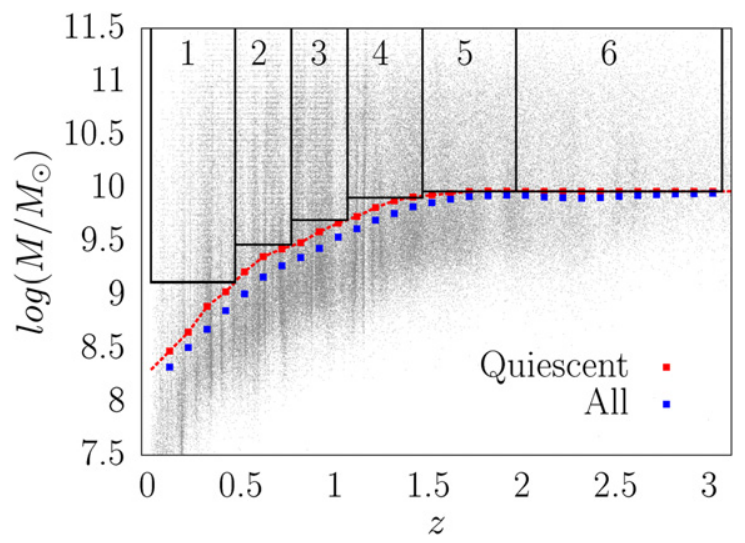

Figure 6. Mass completeness limit for all the galaxies, along with that of the quiescent systems as a function of redshift. The mass completeness limit is defined in such a way that only less than $5 \%$ of galaxies could be missed in the lower mass distribution of the galaxies. Using the mass completeness limits for the quiescent systems, we define six mass complete samples at different redshifts, as shown here with labels 1-6.

Table 5

Properties of Mass Complete Samples Used in this Study

\begin{tabular}{lccc}
\hline \hline $\begin{array}{l}\text { Sample } \\
\text { Number }\end{array}$ & $\begin{array}{c}\text { Redshift } \\
\text { Range }\end{array}$ & $\begin{array}{c}\text { Mass Completeness } \\
\text { Limit } \\
\log \left(M_{\odot}\right)\end{array}$ & $\begin{array}{c}\text { Number of } \\
\text { Galaxies }\end{array}$ \\
\hline 1 & $0.1 \leqslant z<0.5$ & 9.14 & 9338 \\
2 & $0.5 \leqslant z<0.8$ & 9.47 & 11760 \\
3 & $0.8 \leqslant z<1.1$ & 9.70 & 13885 \\
4 & $1.1 \leqslant z<1.5$ & 9.93 & 13640 \\
5 & $1.5 \leqslant z<2.0$ & 9.97 & 12217 \\
6 & $2.0 \leqslant z<3.1$ & 9.97 & 12641 \\
\hline
\end{tabular}

\subsection{Sample Selection}

In the following subsections, we first define a mass complete sample at different redshifts. We also explain the selection of quiescent/star-forming galaxies.

\subsubsection{Stellar Mass Complete Samples}

The magnitude cut $\mathrm{K}_{s}<24$ introduced in Section 2 results in a magnitude-limited sample whose mass completeness is a function of redshift and stellar mass-to-light ratio M/L. In order to estimate the mass completeness of our sample, we use the method explained in Pozzetti et al. (2010) and Ilbert et al. (2013). First, for each galaxy, we calculate the limiting stellar mass $\left(M_{\text {lim }}\right)$, that is, the mass it would have at its redshift if its apparent magnitude were equal to the magnitude limit of the sample $\left(\mathrm{K}_{s}=24\right)$. This is given by $\log \left(M_{\lim } / M_{\odot}\right)=\log \left(M / M_{\odot}\right)+0.4$ $\left(\mathrm{K}_{s}-24\right)$, where $M$ is the estimated stellar mass of the galaxy with apparent magnitude $\mathrm{K}_{s}$. At each redshift, the stellar mass completeness limit $\left(M_{\text {comp }}\right)$ corresponds to the mass with $95 \%$ of the galaxies having their $M_{\text {lim }}$ below the stellar mass completeness limit. This guarantees that only less than $5 \%$ of the galaxies could be missed in the lower mass regime of the stellar mass distribution of galaxies. The mass completeness limit also depends on the stellar $\mathrm{M} / \mathrm{L}$ and is higher for quiescent galaxies. Therefore, in constructing the mass complete samples at each redshift, we make sure that we rely on the quiescent galaxies to estimate the stellar completeness limit. This minimizes the loss of low-mass quiescent galaxies, especially at higher redshifts. The selection of quiescent/star-forming galaxies is explained in 

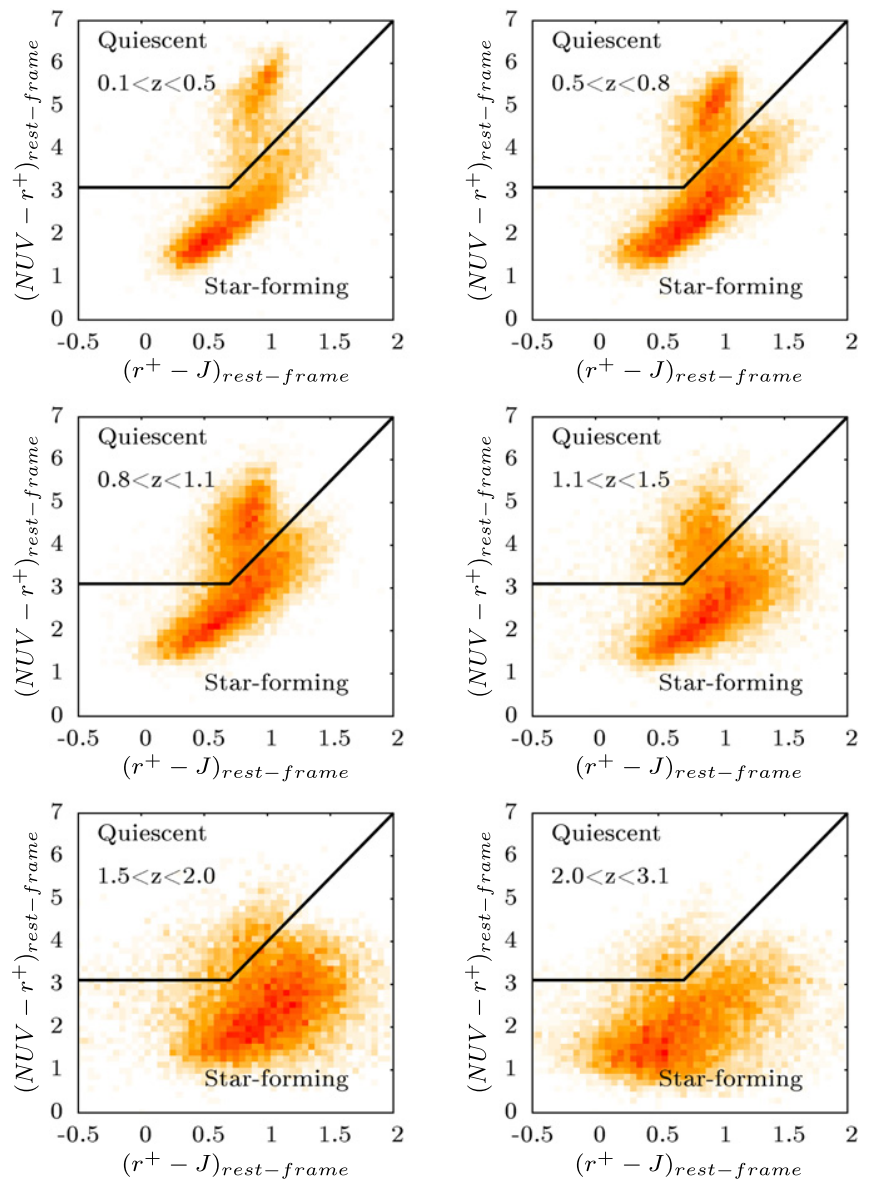

Figure 7. NUV- $r^{+}$vs. $r^{+}-J$ color-color plots used to select quiescent and starforming populations in our mass complete samples at $z=0.1-3.1$. Galaxies with their rest-frame color NUV-r$r^{+}>3.1$ and NUV-r $r^{+}>3\left(r^{+}-J\right)+1$ are selected as quiescent systems (Ilbert et al. 2013).

Section 7.1.2. Figure 6 shows the stellar mass of galaxies as a function of redshift for the magnitude-limited sample $\left(\mathrm{K}_{s}<24\right)$ defined in Section 2. The mass completeness limits for all the galaxies (quiescent and star-forming) and quiescent systems are shown in Figure 6 . As mentioned, only less than 5\% of galaxies could be missed in the lower mass population of galaxies. The mass completeness limit is higher for quiescent systems. Here, we define six mass complete samples out to $z \sim 3$, as shown in Figure 6. The properties of these mass complete samples are given in Table 5.

\subsubsection{Selection of Quiescent/Star-forming Systems}

The selection of quiescent and star-forming systems can be performed using a single rest-frame color through the colormagnitude diagram (CMD). However, the single color selection is problematic for several reasons. The existence of dusty, star-forming galaxies that mimic the color of quiescent systems can significantly contaminate the quiescent population and tends to unrealistically increase the quiescent fraction and their comoving number densities (Brammer et al. 2009). The larger scatter in the rest-frame color at higher redshifts (mostly due to photo- $z$ uncertainties) can wash out the red sequence and the disappearance of the rest-frame color bimodality at $z \gtrsim 1.5$ (Williams et al. 2009). Here, we use rest-frame two-color NUV $-r^{+}$versus $r^{+}-J$ in order to select quiescent and star-

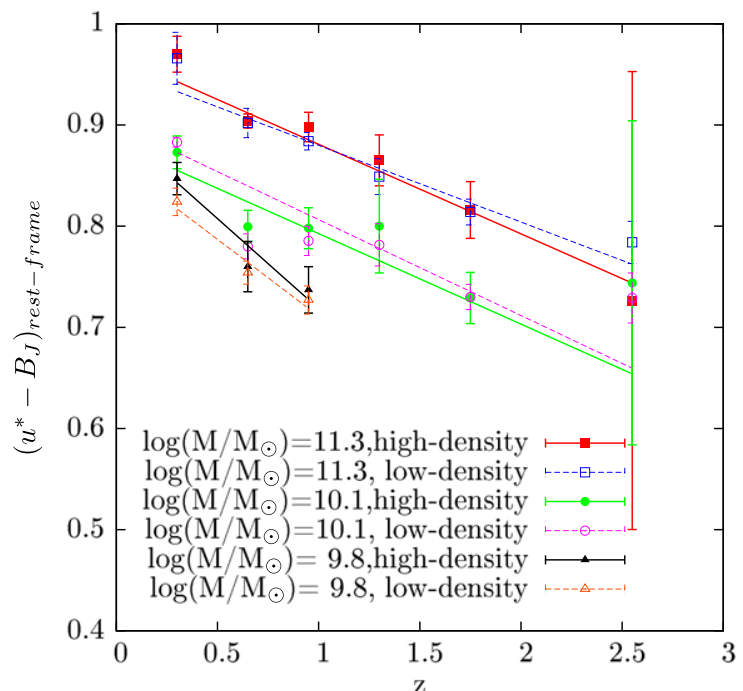

Figure 8. Redshift evolution of the median rest-frame $u^{*}-B_{J}$ color for quiescent galaxies with different stellar masses located in high- and low-density environments. For clarity, the evolution is shown for only a few stellar masses. The color uncertainties are estimated using 10,000 bootstrap resamples added in quadrature to the median observational uncertainties in color. We assume that the evolution of the rest-frame $u^{*}-B_{J}$ color for quiescent systems is linear with redshift, and fit a straight line to the median colors at any given mass and environment. At a fixed redshift and stellar mass, the color of quiescent galaxies is independent of environment. However, at a fixed redshift and environment, the color of quiescent galaxies depends on stellar mass. Quiescent galaxies become redder with cosmic time and their evolution is independent of the environment in which they reside. Quiescent galaxies more massive than $\log \left(M / M_{\odot}\right) \gtrsim 10$ become $\sim 0.2$ mag redder in rest-frame $u^{*}-B_{J}$ color since $z \sim 2.5$. Since $z \sim 1$, less massive systems $\left(\log \left(M / M_{\odot}\right) \sim 9.5-10\right)$ redden by $\sim 0.1 \mathrm{mag}$, regardless of their environment. We also find that more massive quiescent galaxies $\left(\log \left(M / M_{\odot}\right) \sim 11\right)$ are $\sim 0.1$ mag redder in the restframe $u^{*}-B_{J}$ color compared to less massive $\left(\log \left(M / M_{\odot}\right) \sim 10\right)$ systems, and this color difference is almost independent of the environment and redshift.

forming populations in our mass complete samples. It has been shown that the rest-frame NUV- $r^{+}$color is a better indicator of recent star-formation activity (Martin et al. 2007) and has a wider dynamical range compared to the more commonly used rest-frame $U-V$ color (Ilbert et al. 2013). Here, galaxies with a rest-frame color NUV- $r^{+}>3.1$ and $\mathrm{NUV}-r^{+}>3\left(r^{+}-J\right)+1$ are selected as quiescent systems (Ilbert et al. 2013). Figure 7 shows the rest-frame NUV- $r^{+}$versus $r^{+}-J$ distribution of galaxies in the mass complete samples and the color cuts used to separate quiescent and star-forming systems. Two distinct populations of galaxies are clearly seen out to $z \sim 3$. We stress that in our two-color selection technique, adding dust to the star-forming galaxies causes them to move diagonally from the bottom left to the top right of Figure 7, making them separable from the quiescent systems (Williams et al. 2009; Ilbert et al. 2013).

\subsection{Evolution of Rest-frame Color as a Function of Environment For Quiescent Galaxies}

We now investigate the effect of the environment on the restframe $u^{*}-B_{J}$ color of quiescent systems with different stellar masses and its evolution with redshift. We note that in selecting the quiescent population, we do not use the single rest-frame $u^{*}-B_{J}$ color, due to the issues expressed in Section 7.1.2. The selection is alternatively based on the rest-frame NUV $-r^{+}$ versus $r^{+}-J$ plot. Here, we define two environments: the highdensity environment (galaxy group and cluster scales) is 
Table 6

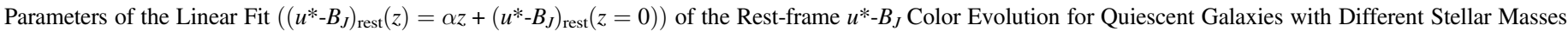

\begin{tabular}{|c|c|c|c|c|}
\hline \multirow{2}{*}{$\begin{array}{l}M \\
\log \left(M_{\odot}\right)\end{array}$} & \multicolumn{2}{|c|}{$\alpha$} & \multicolumn{2}{|c|}{$\left(u^{*}-B_{J}\right)_{\mathrm{rest}}(z=0)$} \\
\hline & High-density & Low-density & High-density & Low-density \\
\hline 11.3 & $-0.089 \pm 0.020$ & $-0.076 \pm 0.010$ & $0.969 \pm 0.016$ & $0.956 \pm 0.012$ \\
\hline 10.8 & $-0.082 \pm 0.032$ & $-0.076 \pm 0.011$ & $0.917 \pm 0.026$ & $0.920 \pm 0.016$ \\
\hline 10.5 & $-0.082 \pm 0.023$ & $-0.065 \pm 0.016$ & $0.916 \pm 0.024$ & $0.882 \pm 0.019$ \\
\hline 10.1 & $-0.089 \pm 0.020$ & $-0.095 \pm 0.022$ & $0.882 \pm 0.018$ & $0.901 \pm 0.018$ \\
\hline 9.8 & $-0.178 \pm 0.041$ & $-0.151 \pm 0.035$ & $0.896 \pm 0.025$ & $0.863 \pm 0.024$ \\
\hline 9.6 & -0.176 & -0.157 & 0.885 & 0.853 \\
\hline
\end{tabular}

defined as regions with overdensity values $\log (1+\delta) \geqslant 0.5$ and the low-density environment (field-like environment) is defined as those with $\log (1+\delta)<0.5$. The selection of the cut between low- and high-density environments is somewhat arbitrary. However, it is shown (B. Darvish et al. 2015, in preparation) that the environmental effects (e.g., the increase in the fraction of quiescent galaxies with overdensity) start to effectively rise at $\log (1+\delta) \gtrsim 0.5$ in the COSMOS field. Throughout this work, we use this cut to separate the density field into low- and high-density environments. Based on this definition, we study the redshift evolution of the rest-frame $u^{*}$ $B_{J}$ color as a function of the environment and stellar mass. Figure 8 shows the redshift evolution of the median rest-frame $u^{*}-B_{J}$ color for quiescent galaxies with different stellar masses located in high- and low-density environments. For clarity, the evolution is shown for only a few stellar mass bins ( $\Delta M= \pm 0.1 \mathrm{dex}$ around the selected masses). We choose the center of the redshift bins (Table 5) to be the redshift of the given points. The median color at each given environment, stellar mass, and redshift is estimated using all quiescent galaxies in that environment, with their redshift located in the redshift range of the mass complete samples and stellar masses within $\Delta M \pm 0.1$ dex of the given stellar mass. The color uncertainties are estimated using 10,000 bootstrap resamples added in quadrature to the median observational uncertainties in color. We assume that the evolution of the rest-frame $u^{*}-B_{J}$ color for quiescent systems is linear with redshift, and fit a straight line to the median colors at any given mass and environment:

$$
\left(u^{*}-B_{J}\right)_{\text {rest }}(z)=\alpha z+\left(u^{*}-B_{J}\right)_{\text {rest }}(z=0) \text {. }
$$

The result of the fit to the median colors for different environments and stellar masses is given in Table 6. According to Figure 8 and Table 6, quiescent galaxies become redder with cosmic time and their evolution is independent of the environment in which they reside. We particularly find that irrespective of environment, quiescent galaxies that are more massive than $\log \left(M / M_{\odot}\right) \gtrsim 10$ become $\sim 0.2$ mag redder since $z \sim 2.5$. This is in agreement with Kriek et al. (2008), who showed a reddening of $\sim 0.25 \mathrm{mag}$ in the rest-frame $U-B$ color for massive $\left(2 \times 10^{11} M_{\odot}\right)$ red galaxies in the field since $z \sim 2.3$. This reddening is also seen in less massive quiescent systems $\left(\log \left(M / M_{\odot}\right)=10-11\right)$ and is independent of environment since $z \sim 3$. Due to the incompleteness in stellar mass, we can not study the color evolution of less massive galaxies $(\log (M /$ $\left.\left.M_{\odot}\right) \lesssim 10\right)$ at $z \gtrsim 1$. However, at $z \lesssim 1$, these systems $(\log (M /$ $\left.\left.M_{\odot}\right) \sim 9.5-10\right)$ redden by $\sim 0.1 \mathrm{mag}$, regardless of their environment.
We also see that at any given redshift out to $z \sim 3$, more massive quiescent systems are redder compared with less massive systems, regardless of their environment. Particularly, we find that more massive quiescent galaxies $\left(\log \left(M / M_{\odot}\right)\right.$ $\sim 11)$ are $\sim 0.1 \mathrm{mag}$ redder in the rest-frame $u^{*}-B_{J}$ color compared with less massive $\left(\log \left(M / M_{\odot}\right) \sim 10\right)$ systems, and this color difference is independent of the environment. The fact that more massive quiescent systems are redder than the less massive ones is a manifestation of the color-magnitude relation $(\mathrm{CMR})$, because the stellar mass is proportional to the luminosity (magnitude) of galaxies. The CMR is seen in both the field (less dense) and cluster (dense) quiescent galaxies, and in the local universe out to higher redshifts (see, e.g., Bower et al. 1992; Blakeslee et al. 2003; Bell et al. 2004; Brammer et al. 2009; Mei et al. 2009; Wilson et al. 2009; Papovich et al. 2010).

For quiescent galaxies, we find that the rest-frame color at a fixed environment depends on stellar mass, but at a fixed mass it is independent of environment. At first glance this seems to contradict studies showing a tight correlation between color and the local density of galaxies (e.g., Balogh et al. 2004). However, the stellar mass also depends strongly on density and color-density relation is actually a manifestation of a more fundamental color-mass relation. When controlled for the stellar mass (or luminosity), the color-density relation becomes independent of the environment (overdensity).

The environmental independence of the color-mass relation is consistent with several previous studies. In the local universe, for example, Balogh et al. (2004) showed that at a fixed luminosity (equivalent to a fixed stellar mass), the mean rest-frame color of red galaxies in the SDSS at $z<0.08$ is nearly independent of their environment. Independently, Baldry et al. (2006) showed that at $z<0.1$, the color-mass distribution of galaxies (red and blue) does not significantly change with environment. The same result is also seen in Hogg et al. (2004), who found the independence of red bulgedominated galaxy colors on the environment in the SDSS at $z<0.12$. Using the SDSS data, Park et al. (2007) showed that when morphology and luminosity (equivalent to stellar mass in our study) are fixed, other physical properties of galaxies, including color, are nearly independent of the local density of the galaxies. They also showed that more luminous galaxies are redder than less luminous systems, and this was independent of the environment. This is consistent with our results. The environmental invariance of color for bright galaxies in the SDSS is also seen in Tanaka et al. (2004).

Several studies at higher redshifts are also consistent with our results. For example, Cassata et al. (2007) showed that at $z \sim 0.7$ in the COSMOS field, the observed CMD for red and early-type galaxies is independent of the local density of 


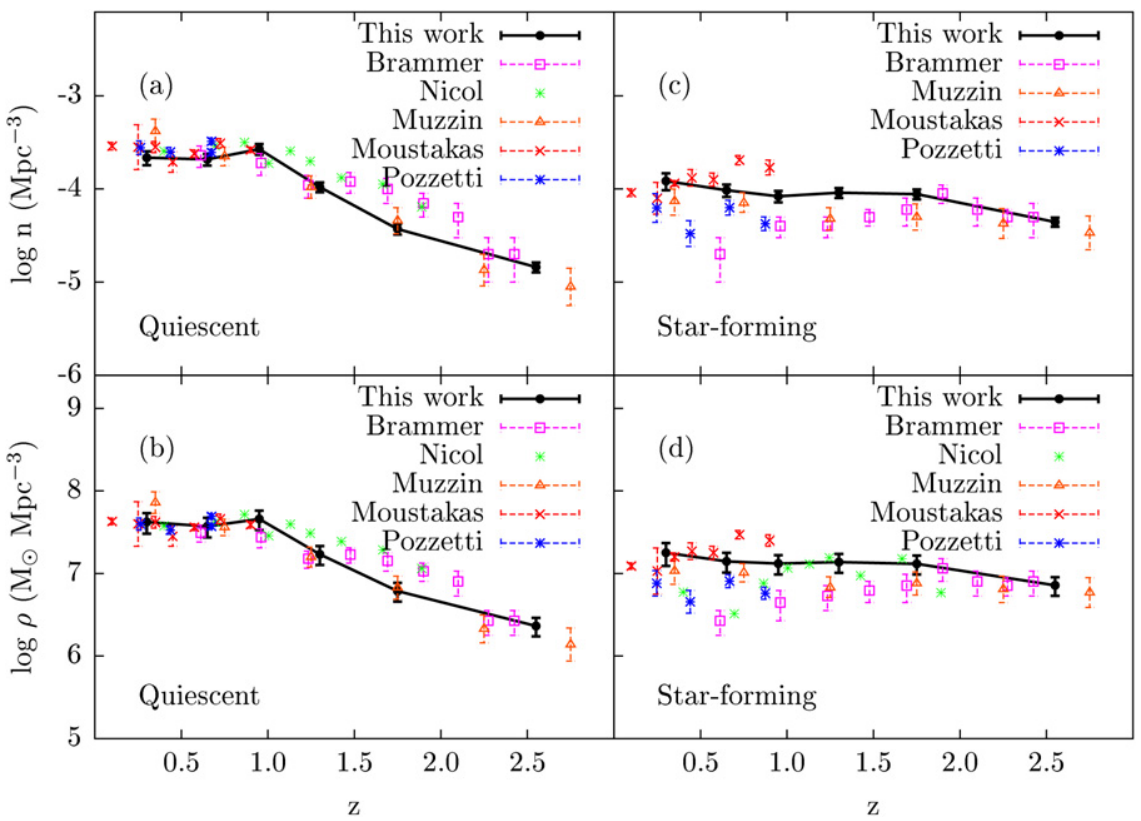

Figure 9. Evolution of comoving number $(n)$ and mass $(\rho)$ density for massive $\left(>10^{11} M_{\odot}\right)$ galaxies (quiescent and star-forming) since $z \sim 3$, along with a comparison with some previous studies. Error bars for number densities incorporate both Poisson error and uncertainties due to the cosmic variance. Uncertainty in mass density is estimated using 10,000 bootstrap resamples, added in quadrature to the observational uncertainties and those due to the cosmic variance. The comoving number and mass density of massive quiescent systems rapidly increase from $z \sim 3$ to $z \sim 1$. However, from $z \sim 1$ to the present time, they remain almost unchanged within the uncertainties. We find almost no evolution in the comoving number and mass density of massive star-forming galaxies since $z \sim 2$. The number and mass density of the massive star-forming galaxies are slightly increased from $z=2.0-3.1$ to $z=1.5-2.0$ (a factor of $\sim 2$ ) and remain almost unaltered since then.

galaxies. Scodeggio et al. (2009) found that for a sample of galaxies in the VVDS survey at $0.2<z<1.4$, color is independent of the local density of galaxies at a fixed stellar mass. Using the $z$ COSMOS data out to $z \sim 1$, Moresco et al. (2010) found that the rest-frame color of red galaxies at a fixed stellar mass is almost independent of environment, but depends on the stellar mass at a fixed environment. They also found that the average colors of massive red galaxies $\left(\log \left(M / M_{\odot}\right)=10.8\right)$ are redder than low-mass galaxies $\left(\left(\log \left(M / M_{\odot}\right)=10\right)\right.$ throughout their entire redshift range. This is entirely consistent with our results in this section. By extending this study to higher redshifts, we demonstrate that the results hold to $z \sim 3$. Cucciati et al. (2010) showed that for red massive galaxies $\left(\left(\log \left(M / M_{\odot}\right) \gtrsim 10.7\right)\right.$ in the $z$ COSMOS $10 \mathrm{k}$-sample, the colordensity relation is globally flat up to $z \sim 1$, which is consistent with our results. Recently, Bassett et al. (2013) compared the rest-frame $U-B$ color of the galaxies in a cluster with those in the field at $z=1.6$ in the CANDELS-UDS field, and found no difference between the color of the quiescent galaxies in these two environments. This agrees well with our results at $z>1$.

\subsection{Effect of the Environment on the Comoving Number and Mass Density of Massive Galaxies}

In this section, we investigate the evolution of comoving number $(n)$ and mass $(\rho)$ density for massive $\left(>10^{11} M_{\odot}\right)$ galaxies (quiescent and star-forming) in different environments since $z \sim 3$. We start our analysis by studying the evolution of $n$ and $\rho$ for massive $\left(>10^{11} M_{\odot}\right)$ quiescent/star-forming systems regardless of their environment. This allows us to compare $n$ and $\rho$ with previous studies that often do not consider the role of environment in the analysis. Later, we discuss the role of environment.

Figure 9 shows the evolution of the comoving number and mass density of massive $\left(>10^{11} M_{\odot}\right)$ quiescent and star- forming galaxies since $z \sim 3$, along with a comparison with some previous studies. Some of these studies use Kroupa IMF to estimate stellar masses. For those, we modify the mass densities based on the Chabrier IMF $\left(M_{\mathrm{Chab}} \sim 0.89 M_{\text {Kroupa }}\right)$. The redshift of our data points is selected as the center of the redshift bins introduced for mass complete samples (Table 5). Error bars for number densities incorporate both Poisson error and uncertainties due to the cosmic variance. Uncertainty in mass density is estimated using 10,000 bootstrap resamples, added in quadrature to the observational uncertainties and those due to the cosmic variance. For observational uncertainties, we use the median of the stellar mass uncertainties of the galaxies in each subsample. We use the cosmic variance calculator of Moster et al. (2011) to estimate the cosmic variance uncertainties. For the $11<\log \left(M / M_{\odot}\right)<11.5$ galaxies, the fractional uncertainties due to the cosmic variance change between $\sim 15-10 \%$ at $\sim z=0.1-3$ in the COSMOS.

\subsubsection{Massive Quiescent Galaxies}

Figures 9(a) and (b) show a rapid increase in the comoving number and mass density of massive quiescent systems from $z \sim 3$ to $z \sim 1$. However, we observe a change of pattern at $z \sim 1$. From $z \sim 1$ to the present time, the number and mass density of massive quiescent galaxies remain almost unchanged within the uncertainties. From $z=2-3.1$ to $z=1.5-2.0$, the number and mass density of massive quiescent galaxies increase by a factor of $\sim 2.5$. From $z=1.5-2.0$ to $z=0.8-1.1$, the rise in the number and mass density of massive quiescent galaxies is steeper and they are increased by a factor of $\sim 6-8$.

The lack of evolution (or insignificant evolution) since $z \sim 1$ in the comoving number and mass density of massive quiescent systems is consistent with several studies. In terms of sample selection, the closest works to our study are those of Ilbert et al. (2013) and Muzzin et al. (2013b). Ilbert et al. (2013) did not 
Table 7

Comoving Number Density, $n$ (in Units of $10^{-4} \mathrm{Mpc}^{-3}$ ). and Mass Density, $\rho$ (in Units of $10^{7} M_{\odot} \mathrm{Mpc}^{-3}$ ), of Massive $\left(>10^{11} M_{\odot}\right)$ Quiescent and Star-forming Galaxies in Different Redshift Bins

\begin{tabular}{lllllr}
\hline \hline Property & \multicolumn{3}{c}{ Redshift } \\
\hline & $0.1 \leqslant z<0.5$ & $0.5 \leqslant z<0.8$ & $0.8 \leqslant z<1.1$ & $1.1 \leqslant z<1.5$ & $1.5 \leqslant z<2.0$ \\
\hline$n_{Q}$ & $2.17 \pm 0.37$ & $2.08 \pm 0.30$ & $2.68 \pm 0.35$ & $1.05 \pm 0.13$ & $0.37 \pm 0.05$ \\
$n_{\mathrm{SF}}$ & $1.22 \pm 0.25$ & $0.97 \pm 0.15$ & $0.84 \pm 0.12$ & $0.91 \pm 0.11$ & $0.88 \pm 0.11$ \\
$\rho_{Q}$ & $4.20 \pm 1.17$ & $3.73 \pm 1.00$ & $4.57 \pm 1.20$ & $1.71 \pm 0.44$ & $0.61 \pm 0.16$ \\
$\rho_{\mathrm{SF}}$ & $1.78 \pm 0.55$ & $1.40 \pm 0.37$ & $1.32 \pm 0.35$ & $1.37 \pm 0.35$ & $0.44 \pm 0.05$ \\
\hline
\end{tabular}

find any significant evolution in the number and mass density of high-mass end quiescent galaxies at $z<1$, which is consistent with our results. As seen in Figures 9(a) and (b), Muzzin et al. (2013b) data points follow our observed trends for $n$ and $\rho$ relatively well, within the uncertainties. As seen in Figures 9(a) and (b), for quiescent galaxies at $z<1$, our result is also in a good agreement with $>10^{11} M_{\odot}$ red-sequence galaxies in the COMBO17 + 4 Survey from Nicol et al. (2011), massive $U-V-J$ selected quiescent galaxies in the NMBS survey from Brammer et al. $(2011), \log \left(M / M_{\odot}\right)=11-11.5$ red galaxies in zCOSMOS from Pozzetti et al. (2010), quiescent systems in the PRIMUS survey from Moustakas et al. (2013), and $>10^{11} M_{\odot}$ rest-frame $(U-V)$ selected red galaxies in VIPERS from Davidzon et al. (2013). Several luminosity function studies at $z<1$, such as Scarlata et al. (2007) and Cool et al. (2008), saw almost no evolution in the number density of very luminous $\left(L \sim 2.5-3 L^{*}\right)$ red or early-type galaxies since $z \sim 1$, which is also in agreement with our results.

The sharp rise in the comoving number and mass density of massive quiescent systems from $z=3-1$ is also in agreement with some previous studies. For example, Ilbert et al. (2013) found that the comoving number and mass density of $10^{11} M_{\odot}$ quiescent galaxies increase by factors of 25 and 13 , respectively, between $2.5<z<3$ and $0.8<z<1.1$. This is similar to our result, which showed an increase of $\sim 15-20$ in $n$ and $\rho$ from $z \sim 3$ to $z \sim 1$ for massive quiescent systems. Our results also agree qualitatively and/or quantitatively with Brammer et al. (2011), Domínguez Sánchez et al. (2011), Nicol et al. (2011), and Muzzin et al. (2013b).

\subsubsection{Massive Star-forming Galaxies}

The situation is different for massive $\left(>10^{11} M_{\odot}\right)$ starforming systems. According to Figures $9(\mathrm{c})$ and (d), we find almost no evolution in the comoving number and mass density of star-forming galaxies since $z \sim 2$. The number and mass density of massive star-forming galaxies are slightly increased from $z=2.0-3.1$ to $z=1.5-2.0$ (a factor of $\sim 2$ ), and remain almost unaltered since then. This slight (insignificant) increase is seen in Brammer et al. (2011) from $z \sim 2.5$ to 2.0, followed by decline since $z \sim 2$. In our study we do not find any sign of decline in the comoving number and mass density of the massive star-forming systems since $z \sim 2 .^{8}$ Our result also agrees well with Pozzetti et al. (2010), Davidzon et al. (2013), Ilbert et al. (2013), Muzzin et al. (2013b), and Sobral et al. (2014). However, Moustakas et al. (2013) found that the number (and mass) density of massive star-forming galaxies are decreased slightly by a factor $\sim 2$ since $z \sim 1$ (Figures 9 (c) and $(\mathrm{d}))$. Within the uncertainties, we do not find a significant

\footnotetext{
8 Brammer et al. (2011) state in their paper that the comoving number density evolution of $>10^{11} M_{\odot}$ star-forming galaxies is nearly flat out to $z=2.0$.
}

disagreement between our result and that of Moustakas et al. (2013), although we detect no evolution in $n$ and $\rho$ for massive star-forming galaxies. We argue that even in the presence of a real evolution in number and mass density of massive starforming systems since $z \sim 1$, we would not be able to observe it due to the larger error bars in our study compared with Moustakas et al. (2013).

The comoving number and mass density values for massive $\left(>10^{11} M_{\odot}\right)$ quiescent/star-forming populations are given in Table 7 .

\subsubsection{Massive Quiescent/Star-Forming Systems in Different Environments}

Now, we investigate the role of the environment in shaping the number and mass density of massive quiescent and starforming galaxies. In order to estimate comoving number and mass densities in different environments, one needs to determine what fraction of the density field is occupied by massive galaxies in different environments. Practically, this is not straightforward because the definition of environment and the selection of low- and high-density regions are arbitrary. We highlight that we only wish to relatively compare the number and mass densities in different environments, rather than determine the exact values in these regions. If there are any systematic errors in determining the number and mass densities in different environments, they would likely be cancelled out because we take the ratio of these in low- and high-density regions. We define the ratio of the comoving number density of massive $\left(>10^{11} M_{\odot}\right)$ galaxies in high-density $\left(n_{\mathrm{hd}}\right)$ to lowdensity $\left(n_{\mathrm{ld}}\right)$ environments as:

$$
\frac{n_{\mathrm{hd}}}{n_{\mathrm{ld}}}=\frac{\sum_{i} 1 / V_{i}^{\mathrm{hd}}}{\sum_{j} 1 / V_{j}^{\mathrm{ld}}},
$$

where $V_{i}^{\text {hd }}$ and $V_{j}^{\text {ld }}$ are the volumes associated with the $i$ th and $j$ th massive galaxy in high- and low-density environments, respectively. The volume assigned to each galaxy is estimated using its Voronoi area $(A)$ and the radial comoving length $(\Delta l)$ that corresponds to its $z$-slice (i.e., $V_{i}=A_{i} \Delta l_{i}$ ). Similarly, we define the ratio of the mass density of massive $\left(>10^{11} M_{\odot}\right)$ galaxies in high-density $\left(\rho_{\mathrm{hd}}\right)$ to low-density $\left(\rho_{\mathrm{ld}}\right)$ environments as:

$$
\frac{\rho_{\mathrm{hd}}}{\rho_{\mathrm{ld}}}=\frac{\sum_{i} M_{i}^{\mathrm{hd}} / V_{i}^{\mathrm{hd}}}{\sum_{j} M_{j}^{\mathrm{ld}} / V_{j}^{\mathrm{ld}}},
$$

where $\mathbf{M}_{i}^{\mathrm{hd}}$ and $\mathbf{M}_{j}^{\mathrm{ld}}$ are the stellar masses associated with the $i$ th and $j$ th massive galaxy in high- and low-density environments, respectively. 
(a)

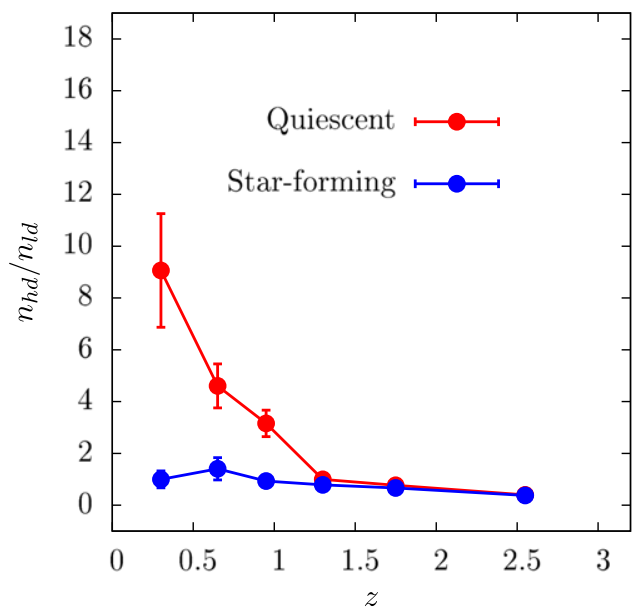

(b)

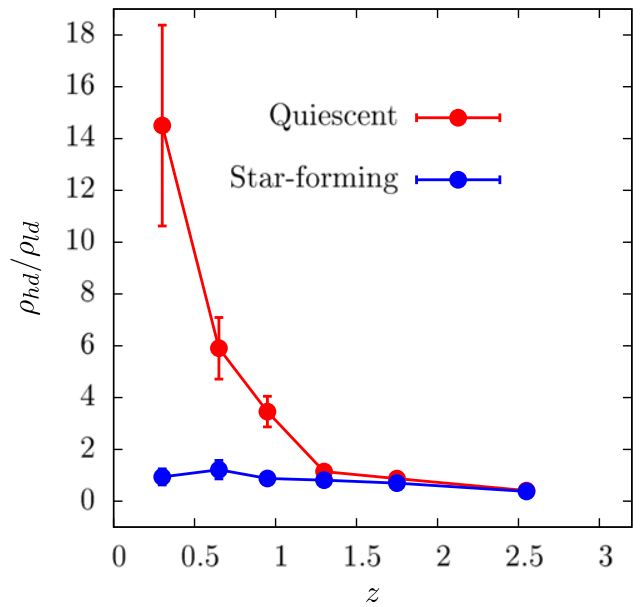

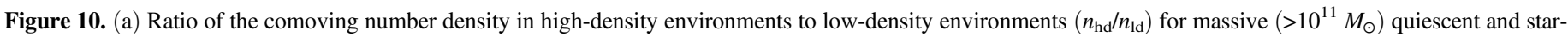

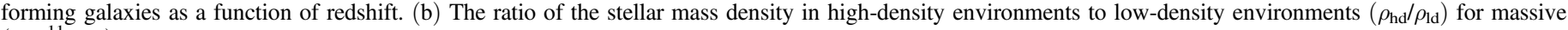

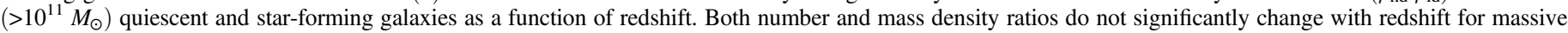

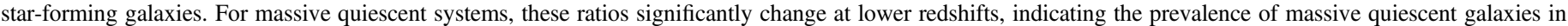
denser environments at lower redshifts.

Figure 10 shows the redshift evolution of $n_{\mathrm{hd}} / n_{\mathrm{ld}}$ and $\rho_{\mathrm{hd}} / \rho_{\mathrm{ld}}$ for massive quiescent and star-forming galaxies. Uncertainties in number density ratios incorporate both Poisson error and the cosmic variance. The error bars for mass density ratios are estimated using 10,000 bootstrap resamples added in quadrature to the observational uncertainties in stellar mass and those due to the cosmic variance. For massive $\left(>10^{11} M_{\odot}\right)$ starforming systems, the number and mass densities in the different environments remain almost the same, and we find almost no evolution in the comoving number and mass density ratios in different environments with cosmic time. Massive star-forming galaxies populate dense and less-dense regions almost equally, regardless of their redshift. The situation is different for massive quiescent galaxies. At $z \gtrsim 1.3$, there is no significant evolution in the comoving number and mass density ratios in different environments. At these redshifts, the number and mass density ratios for massive quiescent galaxies are almost equal to those of massive star-forming systems. However, at lower redshifts $(z \lesssim 1.3)$, we find a significant evolution in the comoving number and mass density ratios for massive quiescent systems in different environments. These ratios for massive quiescent galaxies monotonically increase with cosmic time at $z \lesssim 1.3$. At $z \lesssim 0.5$, the number and mass density of massive quiescent galaxies are $\sim 1$ dex higher in denser regions compared with less-dense environments. Dense environments at low redshifts are populated by massive quiescent galaxies.

We stress that part of the evolution in the comoving number and mass density of massive quiescent systems in different environments may be due to the growth of the large-scale structure with cosmic time. As time progresses, less-dense environments eventually coalesce to assemble more massive, denser regions. We also mention that the lack of evolution at higher redshifts in the number and mass density ratios for massive quiescent galaxies in different environments does not necessarily mean that the environment at higher redshifts is not able to quench the star-formation activity in galaxies as effectively as it does at lower redshifts. The likelihood of finding massive halos that are able to suppress the starformation activity at higher redshifts is low, given the small size of the COSMOS field and the rarity of massive halos at higher redshifts. If we could numerously find such dense massive halos at higher redshifts in our survey, we might be able to see similar environmental trends to those we observe in lower redshifts and the local universe.

It is challenging to make a direct, quantitative comparison between our results in this section and similar studies in the literature, due to the differences in sample selections, the definition of environment, and the classification of low- and high-density regions. However, we qualitatively compare our results in this section with some similar studies. Using $z$ COSMOS data at $z<1$, Bolzonella et al. (2010) studied the galaxy stellar mass function (SMF) as a function of galaxy type and environment. They found that massive galaxies are preferentially located in high-density environments, which are characterized on average by a higher $M^{*}$, and that the spectraltype selected early-type galaxies dominate the high-mass (log $\left.\left(M / M_{\odot}\right) \gtrsim 10.7\right)$ end of the SMF. In other words, massive red galaxies preferentially reside in high-density regions at $z \lesssim 1$, which is in agreement with our results for massive, quiescent galaxies in dense environments. Recently, Mortlock et al. (2015) studied the SMF of galaxies in the UKIDSS/UDS and CANDELS fields, and found that at higher redshifts $(z>1)$ the SMF parameters for galaxies in low and high densities are almost the same. However, at $z<1$, they found evidence that (1) the high-mass end of the galaxy SMF is more dominated by galaxies in dense environments. (2) The high-mass end of the Sérsic index $>2.5 \mathrm{SMF}$ is dominated by quiescent galaxies. (3) On average, $\phi^{*}$ and $M^{*}$ values of the SMF are larger for red galaxies in denser regions compared with those in less-dense environments (at $z \sim 0.5, \phi^{*}$ is $\sim 1$ dex larger for red galaxies in denser regions, which is similar to our result in this section). Combining these results, we conclude that at lower redshifts high-density environments cause the build-up of high-mass, quiescent galaxies. This is completely consistent with our results in this section regarding the higher number and mass densities in denser regions for massive, quiescent systems at lower redshifts, as well as the lack of any significant differences at higher redshifts. 
We stress that our results here rely on the density field estimation based on the flux-limited sample $\left(\mathrm{K}_{s}<24\right)$ introduced in Section 2. However, as we discussed in Section 7.1.1, this magnitude cut results in a sample with varying stellar mass as a function of redshift. In other words, the "typical" galaxies defining the environment change with redshift, with less massive galaxies defining the low $-z$ and more massive systems defining the high- $z$ environments. Because there is some degree of correlation between the galaxy stellar mass and environment (Kauffmann et al. 2004; Baldry et al. 2006), our results in this section may be affected by the selection of galaxies used to estimate the density field (environment). However, in the appendix, we investigate the role of different samples used for density estimation on the results, and will show that the results do not change (they even become stronger) and we recover the same trends discussed in this section.

We conclude that the comoving number and mass density of massive, star-forming systems do not evolve much with redshift, regardless of their environment. This scenario is also true for massive, quiescent galaxies at higher redshifts $(z \gtrsim 1.3)$. However, at lower redshifts, the comoving number and mass density of massive, quiescent galaxies are greater in high-density environments compared with less-dense regions. This highlights the significant role of the environment in quenching the star-formation activity in galaxies at lower redshifts.

\section{SUMMARY AND CONCLUSIONS}

In this work, we used a $\mathrm{K}_{s}$-band selected sample of galaxies with accurate photometric redshifts in COSMOS at $z=0.1-3.1$ in order to estimate the density field. The density field was determined using the weighted versions of the adaptive kernel smoothing, tenth and fifth NN, Voronoi tessellation, and Delaunay triangulation methods. We evaluated the performance of each density estimator using extensive realistic and Monte-Carlo simulations. We later defined two environments and studied the effects of the environment on the mass complete sample of quiescent and star-forming galaxies out to $z \sim 3$. The rest-frame NUV-r ${ }^{+}-J$ color-color plots were used to separate the galaxy population into quiescent and star-forming systems. We investigated the redshift evolution of the restframe $u^{*}-B_{J}$ color for quiescent galaxies as a function of environment and stellar mass. We also studied the evolution of the comoving number and mass density of massive quiescent and star-forming galaxies, as well as their dependence on the environment of the galaxies. Our main results are as follows.

1. We find an overall good agreement between the density field estimated with weighted versions of adaptive kernel smoothing, NN, Voronoi tessellation, and Delaunay triangulation methods over $\sim 2$ orders of magnitude.

2. Extensive simulations show that the adaptive kernel smoothing and Voronoi tessellation outperform other methods in estimating the density field of the galaxies. We recommend using these estimators as a more reliable and robust substitute for the widely used NN or count-inaperture methods.

3. At fixed stellar mass, the median rest-frame $u^{*}-B_{J}$ color of quiescent galaxies is independent of the environment in which they reside. Quiescent galaxies become redder with cosmic time and their color evolution is independent of their environment. Since $z \sim 3$, more massive quiescent galaxies $\left(\log \left(M / M_{\odot}\right) \gtrsim 10\right)$ have become $\sim 0.2$ mag redder in the rest-frame $u^{*}-B_{J}$, whereas less massive quiescent systems have reddened by $\sim 0.1 \mathrm{mag}$ since $z \sim 1$.

4. On average, more massive quiescent galaxies are redder compared to less massive ones at any given redshift, regardless of their environment. The lack of a correlation between color and environment at fixed stellar mass for quiescent galaxies suggests that the relation between stellar mass and the local density of the galaxies is more fundamental than the color-density relation.

5. The average comoving number and mass density of massive $\left(\log \left(M / M_{\odot}\right)>11\right)$ star-forming galaxies have not evolved much since $z \sim 3$. However, for massive quiescent galaxies, the number and mass densities rise sharply from $z \sim 3$ to $z \sim 1$, and remain almost unchanged since then.

6. The evolution of the comoving number and mass density of massive star-forming galaxies do not depend on their environment. They remain almost unchanged since $z \sim 3$, regardless of their host environment. The situation is different for massive quiescent galaxies. The comoving number and mass density of massive quiescent galaxies do not change much with environment and redshift from $z \sim 3$ to $z \sim 1.3$, which is similar to those of star-forming galaxies. However, at lower redshifts $(z \lesssim 1.3)$, we find a significant evolution in the number and mass density of massive quiescent galaxies in denser environments compared with less-dense regions. Dense environments at lower redshifts are populated with massive quiescent galaxies, which signifies the role of the environment in quenching the star-formation activity in galaxies at lower redshifts.

This paper is the first in a series and provided the required tools for the density-based environmental study of galaxies. In a following paper (in preparation), we will study the effects of the local environment of galaxies on their SFR, sSFR, and restframe color, as well as the fraction of quiescent/star-forming systems as a function of redshift, stellar mass, and galaxy type. We will also discuss the fractional role of the stellar mass and environment in suppressing the star-formation activity in galaxies.

We gratefully thank the anonymous referee for thoroughly reading the original manuscript and providing very useful comments that improved the quality of the work. B.D. gratefully acknowledges Lucia Pozzetti for providing the data for this study. D.S. acknowledges financial support from LKBF, the Netherlands Organisation for Scientific research (NWO) through a Veni fellowship, from FCT through an FCT Investigator Starting Grant, a Start-up Grant (IF/01154/2012/ CP0189/CT0010), and the grant PEst-OE/FIS/UI2751/2014. This study used the COSMOS data based on observations with the NASA/ESA Hubble Space Telescope, obtained at the Space Telescope Science Institute, which is operated by AURA Inc., under NASA contract NAS 5-26555, and the Spitzer Space Telescope, which is operated by the Jet Propulsion Laboratory, California Institute of Technology under NASA contract 1407. It is also based on data collected at the Subaru Telescope, which is operated by the National Astronomical Observatory of Japan; XMM-Newton, an ESA science mission with instruments and contributions directly funded by ESA Member 
(a)

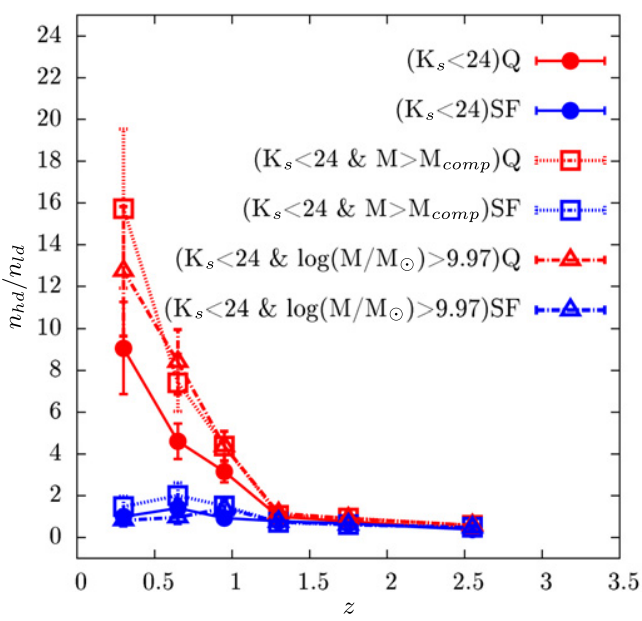

(b)

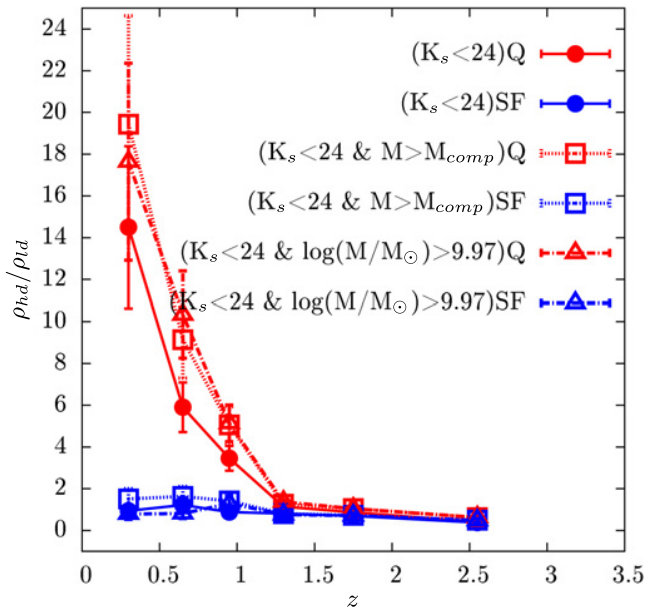

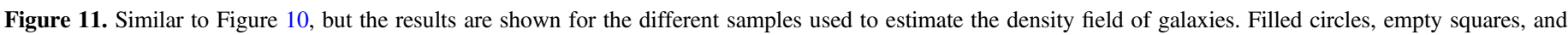

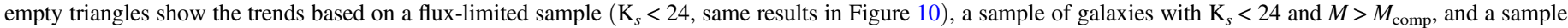

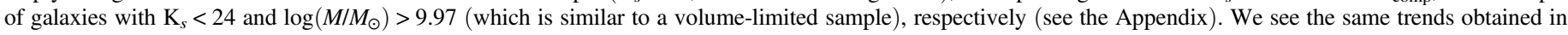
Section 7.3.3. Our results are not significantly influenced by the selection of the samples used to estimate the density field of galaxies.

States and NASA; the European Southern Observatory under Large Program 175.A-01279, Chile; Kitt Peak National Observatory, Cerro Tololo Inter-American Observatory, and the National Optical Astronomy Observatory, which are operated by the Association of Universities for Research in Astronomy, Inc. (AURA) under cooperative agreement with the National Science Foundation; the National Radio Astronomy Observatory, which is a facility of the National Science Foundation operated under cooperative agreement by Associated Universities, Inc.; and the Canada-France-Hawaii Telescope with MegaPrime/MegaCam operated as a joint project by the CFHT Corporation, CEA/DAPNIA, the NRC and CADC of Canada, the CNRS of France, TERAPIX, and the University of Hawaii.

\section{APPENDIX \\ EFFECT OF DENSITY FIELD ESTIMATION BASED ON DIFFERENT SAMPLE SELECTIONS}

In Section 2, we used a flux-limited $\left(\mathrm{K}_{s}<24\right)$ sample of galaxies in order to estimate the density field. All the main results presented in Sections 7.2 and 7.3.3 were based on this flux-limited sample. Here, we try two other samples of galaxies to estimate the density field and investigate their effects on the results presented in Section 7.3.3. Just as before, we use the Voronoi tessellation method as the density estimator and the definition of low- and high-density environments given in Section 7.2. These new samples are defined as follows.

1. In addition to the conditions given in Section 2, we select all galaxies that are more massive than the stellar mass completeness limit of the samples defined in Section 7.1 .1 and Table $5\left(\mathrm{~K}_{s}<24\right.$ and $\left.M>M_{\text {comp }}\right)$.

2. In addition to the conditions given in Section 2, we select all galaxies that are more massive than the stellar mass completeness limit of the highest redshift sample defined in Section 7.1.1 and Table $5\left(\mathrm{~K}_{s}<24\right.$ and $\log \left(M / M_{\odot}\right)$ $>9.97)$. This is similar to a volume-limited sample of galaxies.

Using these new samples, we re-estimate the density fields and reinvestigate the results in Section 7.3.3. Figure 11 is similar to Figure 10, but it also shows the results based on the new samples used to estimate the density field. According to Figure 11, we are able to retrieve the main trends obtained in Section 7.3.3 for quiescent (Q) and star-forming (SF) galaxies. However, we mention that for massive quiescent galaxies, the number and mass density trends become even more amplified at $z<1$ when we use the new samples for density estimation. This is expected because the new samples target more massive galaxies in order to estimate the density field, which tend to be more strongly clustered compared with less massive systems. We highlight that our results in Section 7.3.3 are not significantly affected by the selection of the samples we used for density field estimation.

\section{REFERENCES}

Baldry, I. K., Balogh, M. L., Bower, R. G., et al. 2006, MNRAS, 373, 469 Balogh, M. L., Baldry, I. K., Nichol, R., et al. 2004, ApJL, 615, L101 Bamford, S. P., Nichol, R. C., Baldry, I. K., et al. 2009, MNRAS, 393, 1324 Bassett, R., Papovich, C., Lotz, J. M., et al. 2013, ApJ, 770, 58

Bell, E. F., Wolf, C., Meisenheimer, K., et al. 2004, ApJ, 608, 752 Bernardeau, F., \& van de Weygaert, R. 1996, MNRAS, 279, 693 Blakeslee, J. P., Franx, M., Postman, M., et al. 2003, ApJL, 596, L143 Blanton, M. R., Eisenstein, D., Hogg, D. W., \& Zehavi, I. 2006, ApJ, 645, 977 Böhringer, H., Schuecker, P., Guzzo, L., et al. 2004, A\&A, 425, 367 Böhringer, H., Voges, W., Huchra, J. P., et al. 2000, ApJS, 129, 435 Bolzonella, M., Kovač, K., Pozzetti, L., et al. 2010, A\&A, 524, A76 Bond, J. R., Kofman, L., \& Pogosyan, D. 1996, Natur, 380, 603 Bower, R. G., Lucey, J. R., \& Ellis, R. S. 1992, MNRAS, 254, 589 Brammer, G. B., Whitaker, K. E., van Dokkum, P. G., et al. 2009, ApJL, 706, L173

Brammer, G. B., Whitaker, K. E., van Dokkum, P. G., et al. 2011, ApJ, 739, 24 Capak, P., Abraham, R. G., Ellis, R. S., et al. 2007a, ApJS, 172, 284 Capak, P., Aussel, H., Ajiki, M., et al. 2007b, ApJS, 172, 99

Cassata, P., Guzzo, L., Franceschini, A., et al. 2007, ApJS, 172, 270 Chiang, Y.-K., Overzier, R., \& Gebhardt, K. 2014, ApJL, 782, L3 Colless, M., Dalton, G., Maddox, S., et al. 2001, MNRAS, 328, 1039 Cool, R. J., Eisenstein, D. J., Fan, X., et al. 2008, ApJ, 682, 919 Cooper, M. C., Newman, J. A., Coil, A. L., et al. 2007, MNRAS, 376, 1445 Cooper, M. C., Newman, J. A., Madgwick, D. S., et al. 2005, ApJ, 634, 833 Coppin, K. E. K., Geach, J. E., Webb, T. M. A., et al. 2012, ApJL, 749, L43 Cucciati, O., Iovino, A., Kovač, K., et al. 2010, A\&A, 524, A2 Darvish, B., Sobral, D., Mobasher, B., et al. 2014, ApJ, 796, 51 Davidzon, I., Bolzonella, M., Coupon, J., et al. 2013, A\&A, 558, A23 Dietrich, J. P., Erben, T., Lamer, G., et al. 2007, A\&A, 470, 821 
Domínguez Sánchez, H., Pozzi, F., Gruppioni, C., et al. 2011, MNRAS, 417, 900

Dressler, A. 1980, ApJ, 236, 351

Driver, S. P., Hill, D. T., Kelvin, L. S., et al. 2011, MNRAS, 413, 971

Ebeling, H., Edge, A. C., Bohringer, H., et al. 1998, MNRAS, 301, 881

Ebeling, H., Edge, A. C., \& Henry, J. P. 2001, ApJ, 553, 668

Ebeling, H., \& Wiedenmann, G. 1993, PhRvE, 47, 704

Fadda, D., Biviano, A., Marleau, F. R., Storrie-Lombardi, L. J., \& Durret, F. 2008, ApJL, 672, L9

Finoguenov, A., Guzzo, L., Hasinger, G., et al. 2007, ApJS, 172, 182

Gavazzi, R., \& Soucail, G. 2007, A\&A, 462, 459

Geach, J. E., Ellis, R. S., Smail, I., Rawle, T. D., \& Moran, S. M. 2011, MNRAS, 413, 177

George, M. R., Leauthaud, A., Bundy, K., et al. 2011, ApJ, 742, 125

Gioia, I. M., Henry, J. P., Maccacaro, T., et al. 1990, ApJL, 356, L35

Gladders, M. D., \& Yee, H. K. C. 2000, AJ, 120, 2148

Gladders, M. D., \& Yee, H. K. C. 2005, ApJS, 157, 1

Goto, T., Sekiguchi, M., Nichol, R. C., et al. 2002, AJ, 123, 1807

Hao, J., McKay, T. A., Koester, B. P., et al. 2010, ApJS, 191, 254

Hogg, D. W., Blanton, M. R., Brinchmann, J., et al. 2004, ApJL, 601, L29

Ilbert, O., Capak, P., Salvato, M., et al. 2009, ApJ, 690, 1236

Ilbert, O., McCracken, H. J., le Fèvre, O., et al. 2013, A\&A, 556, A55

Kauffmann, G., White, S. D. M., Heckman, T. M., et al. 2004, MNRAS, 353,713

Knobel, C., Lilly, S. J., Iovino, A., et al. 2009, ApJ, 697, 1842

Knobel, C., Lilly, S. J., Iovino, A., et al. 2012, ApJ, 753, 121

Koester, B. P., McKay, T. A., Annis, J., et al. 2007, ApJ, 660, 239

Kovač, K., Lilly, S. J., Cucciati, O., et al. 2010, ApJ, 708, 505

Kriek, M., van der Wel, A., van Dokkum, P. G., Franx, M., \& Illingworth, G. D. 2008, ApJ, 682, 896

Mahajan, S., Raychaudhury, S., \& Pimbblet, K. A. 2012, MNRAS, 427, 1252

Marriage, T. A., Acquaviva, V., Ade, P. A. R., et al. 2011, ApJ, 737, 61

Martin, D. C., Wyder, T. K., Schiminovich, D., et al. 2007, ApJS, 173, 342

Massey, R., Rhodes, J., Ellis, R., et al. 2007, Natur, 445, 286

McCracken, H. J., Milvang-Jensen, B., Dunlop, J., et al. 2012, A\&A, 544, A156

Mehrtens, N., Romer, A. K., Hilton, M., et al. 2012, MNRAS, 423, 1024

Mei, S., Holden, B. P., Blakeslee, J. P., et al. 2009, ApJ, 690, 42

Miller, C. J., Nichol, R. C., Reichart, D., et al. 2005, AJ, 130, 968

Miyazaki, S., Hamana, T., Ellis, R. S., et al. 2007, ApJ, 669, 714

Mobasher, B., Capak, P., Scoville, N. Z., et al. 2007, ApJS, 172, 117
Moresco, M., Pozzetti, L., Cimatti, A., et al. 2010, A\&A, 524, A67

Mortlock, A., Conselice, C. J., Hartley, W. G., et al. 2015, MNRAS, 447, 2

Moster, B. P., Somerville, R. S., Newman, J. A., \& Rix, H.-W. 2011, ApJ, 731,113

Moustakas, J., Coil, A. L., Aird, J., et al. 2013, ApJ, 767, 50

Muzzin, A., Marchesini, D., Stefanon, M., et al. 2013b, ApJ, 777, 18

Muzzin, A., Wilson, G., Demarco, R., et al. 2013a, ApJ, 767, 51

Muzzin, A., Wilson, G., Yee, H. K. C., et al. 2012, ApJ, 746, 188

Nicol, M.-H., Meisenheimer, K., Wolf, C., \& Tapken, C. 2011, ApJ, 727,51

Papovich, C., Momcheva, I., Willmer, C. N. A., et al. 2010, ApJ, 716, 1503

Park, C., Choi, Y.-Y., Vogeley, M. S., et al. 2007, ApJ, 658, 898

Peng, Y.-J., Lilly, S. J., Kovač, K., et al. 2010, ApJ, 721, 193

Pintos-Castro, I., Sánchez-Portal, M., Cepa, J., et al. 2013, A\&A, 558, A100

Planck Collaboration, Ade, P. A. R., Aghanim, N., et al. 2011, A\&A, 536, A8

Platen, E., van de Weygaert, R., Jones, B. J. T., Vegter, G., \& Calvo, M. A. A. 2011, MNRAS, 416, 2494

Porter, S. C., Raychaudhury, S., Pimbblet, K. A., \& Drinkwater, M. J. 2008, MNRAS, 388, 1152

Pozzetti, L., Bolzonella, M., Zucca, E., et al. 2010, A\&A, 523, A13

Reichardt, C. L., Stalder, B., Bleem, L. E., et al. 2013, ApJ, 763, 127

Scarlata, C., Carollo, C. M., Lilly, S. J., et al. 2007, ApJS, 172, 494

Schaap, W. E., \& van de Weygaert, R. 2000, A\&A, 363, L29

Scodeggio, M., Vergani, D., Cucciati, O., et al. 2009, A\&A, 501, 21

Scoville, N., Arnouts, S., Aussel, H., et al. 2013, ApJS, 206, 3

Scoville, N., Aussel, H., Benson, A., et al. 2007a, ApJS, 172, 150

Scoville, N., Aussel, H., Brusa, M., et al. 2007b, ApJS, 172,

Silverman, B. W. 1986, Density Estimation for Statistics and Data Analysis (London: Chapman and Hall)

Sobral, D., Best, P. N., Smail, I., et al. 2011, MNRAS, 411, 675

Sobral, D., Best, P. N., Smail, I., et al. 2014, MNRAS, 437, 3516

Springel, V., White, S. D. M., Jenkins, A., et al. 2005, Natur, 435, 629

Tanaka, M., Goto, T., Okamura, S., Shimasaku, K., \& Brinkmann, J. 2004, AJ, 128, 2677

Tran, K.-V. H., Saintonge, A., Moustakas, J., et al. 2009, ApJ, 705, 809 Vogelsberger, M., Genel, S., Springel, V., et al. 2014, Natur, 509, 177

Williamson, R., Benson, B. A., High, F. W., et al. 2011, ApJ, 738, 139

Williams, R. J., Quadri, R. F., Franx, M., van Dokkum, P., \& Labbé, I. 2009, ApJ, 691, 1879

Wilson, G., Muzzin, A., Yee, H. K. C., et al. 2009, ApJ, 698, 1943

York, D. G., Adelman, J., Anderson, J. E., Jr, et al. 2000, AJ, 120, 1579 